\title{
Neuroimaging of Real-world Audio-visual Sensory Integration in High-functioning Autism
}

\author{
Paula J. Webster \\ West Virginia University, pjwebster@hsc.wvu.edu
}

Follow this and additional works at: https://researchrepository.wvu.edu/etd

Part of the Systems Neuroscience Commons

\section{Recommended Citation}

Webster, Paula J., "Neuroimaging of Real-world Audio-visual Sensory Integration in High-functioning Autism" (2020). Graduate Theses, Dissertations, and Problem Reports. 7656.

https://researchrepository.wvu.edu/etd/7656

This Dissertation is protected by copyright and/or related rights. It has been brought to you by the The Research Repository @ WVU with permission from the rights-holder(s). You are free to use this Dissertation in any way that is permitted by the copyright and related rights legislation that applies to your use. For other uses you must obtain permission from the rights-holder(s) directly, unless additional rights are indicated by a Creative Commons license in the record and/ or on the work itself. This Dissertation has been accepted for inclusion in WVU Graduate Theses, Dissertations, and Problem Reports collection by an authorized administrator of The Research Repository @ WVU.

For more information, please contact researchrepository@mail.wvu.edu. 


\title{
NEUROIMAGING OF REAL-WORLD AUDIO-VISUAL SENSORY INTEGRATION IN HIGH-FUNCTIONING AUTISM
}

PAULA J. WEBSTER, B.A.

Dissertation submitted to the

School of Medicine

at West Virginia University

in partial fulfillment of the requirements for the degree of

DOCTOR OF PHILOSOPHY

in

Neuroscience

\author{
James W. Lewis, Ph.D., Advisor \\ Marc W. Haut, Ph.D., Committee Chair \\ Mary Beth Mandich, Ph.D. \\ Carla Mazefsky, Ph.D. \\ Bernard G. Schreurs, Ph.D.
}

Department of Neuroscience

Morgantown, West Virginia

2020

Keywords: autism spectrum disorder, putamen, globus pallidus, intraparietal sulcus, lentiform nuclei, hypoactivation, attention, functional magnetic resonance imaging, sensory processing

Copyright 2020, Paula J. Webster 


\begin{abstract}
NEUROIMAGING OF REAL-WORLD AUDIO-VISUAL SENSORY INTEGRATION IN HIGH-FUNCTIONING AUTISM
\end{abstract}

\title{
Paula J. Webster
}

Sensory processing differences are a prevalent aspect of autism spectrum disorder (ASD) that may contribute to core deficits of ASD such as repetitive behaviors as well as comorbidities including anxiety disorders. The ability to integrate information among our senses is required to comprehend the world around us and is crucial for the development of language, motor skills, and social communication. Prior studies have shown that individuals with autism differ from individuals without autism when presented with simple, non-natural audio-visual stimuli such as basic shapes accompanied by pure tones. Because the human brain processes non-natural and natural stimuli differently, more recent studies have used real-world images paired with a sound. However, the stimuli used in many of these studies were static photos paired with a congruent sound and do not reflect the dynamic nature of a real-world environment. The bulk of studies using dynamic real-world stimuli have investigated language processing by pairing human vocalizations with a human face. However, because face and vocalization stimuli are processed in distinct areas of the brain, dynamic stimuli that contain faces and vocalizations confound investigations of multisensory integration. The remaining studies that used dynamic real-world videos to investigate multisensory integration, have primarily used very short video clips of only a few seconds in length. While these stimuli do represent the natural environment, the short length 
of these videos lacks the continuous nature of what we see and hear in our environment. Only two studies have used dynamic, real-world stimuli that are continuous to investigate multisensory integration in autism. Unfortunately, the stimuli used in both of these studies contained confounding facial and or language processing. Therefore, we currently do not have a good understanding of how individuals with autism integrate multiple real-world sensory inputs that reflect dynamic natural stimuli encountered in the environment outside of our understanding of language and face processing.

Considering the gap in the current literature regarding processing of realworld, dynamic stimuli, the goal of this research was to use functional magnetic resonance imaging (fMRI) to investigate how individuals with autism integrate auditory and visual information of a real-world, dynamic scene. We hypothesized that individuals with high-functioning autism would show different levels of brain activation in regions known to process auditory and visual information as well as in brain areas known to integrate audio-visual information. While undergoing an MRI, participants (ASD n=20 and typically developed controls [TD] $n=21$ ) watched a video of a person bouncing a basketball. The person was filmed from the neck down to avoid engaging face processing brain regions while viewing the video. To ensure engagement, a simple attention task was used and was easily accomplished by both groups. Analysis of the fMRI data showed that the ASD group had significantly less brain activation in left-lateralized intraparietal sulcus and putamen/globus pallidus. These brain regions are known to be involved in processing human biological motion and regulating motor movements 
respectively. The hypoactivation seen in the ASD group may reflect underconnectivity between and within the hemispheres for processing this dynamic audio-visual stimulus. These data support the Underconnectivity Theory of Autism which posits long-distance networks are underconnected in individuals with autism contributing to global processing deficits. 


\section{DEDICATION}

This work is dedicated to my family. They have inspired me to pursue my passion to conduct autism research and to take on the tremendous task of obtaining my Ph.D. Without their love, patience, sacrifice, and continuing support, this life accomplishment would never have been possible. I am eternally grateful.

This work is also dedicated to the memory of my grandparents Betty and Floyd Breeden and Dorothy and Jim Baker as well as to my in-laws Judy and Gary Ferrel. I never could have imagined you would not be here to see me finish. My accomplishment is your accomplishment. 


\section{ACKNOWLEDGEMENTS}

First and foremost, I greatly appreciate the time and dedication of the participants and their families without whom this research would not have been possible. It has been my great pleasure to get to know you all. Thank you for your dedication to autism research!

I want to acknowledge the love and support from my dear friends. You have provided me with invaluable words of encouragement, shoulders to cry on, incalculable gallons of coffee and other various beverages, along with sound advice. The support of long-time friends has been uplifting and the acquisition of new friendships has by far been the most valuable thing I have attained over these many years at $\mathrm{WVU}$ !

To my lab mates over the years and my PI, Jim Lewis, I am thankful for the opportunity to conduct the first human autism neuroimaging research study at WVU during a time when we did not have a research-dedicated MRI nor the funds to conduct this research. Chris Frum was especially giving of his time and taught me a great deal about fMRI data processing and the inner workings of a lab - the knowledge I gained as a result has proven to be invaluable.

I will always be grateful to the mentors who have provided me with professional guidance throughout my journey. I appreciate the time and guidance my committee members have provided that enabled me to succeed. Dr. Marc Haut's contributions to my writing and progression through the end of my $\mathrm{PhD}$ was invaluable and I am eternally grateful for his encouraging words and the time he has taken to enable me to complete this life goal. I would also like to acknowledge 
mentors outside of my committee. Dr. Tom Marshall has not only provided continuous moral support, but significant contributions to the editing of this document as well. Dr. William Stauber's periodic check-ins and support meant the world to me. Dr. Pete Mathers advice and encouragement in my final years at WVU enabled me to keep my sanity. Finally, Dr. Shuo Wang, from whom I have learned a great deal while working on various autism projects and I deeply appreciate his support and guidance.

I would like to acknowledge the resource contributions of Vanderbilt University, West Liberty University, Marshall University, and the WVU Carruth Center. Funding for this project was provided by a grant from the West Virginia Clinical and Translational Science Institute (NIH/NIGMS Award Number 5U54GM104942-03) based on my PhD research proposal. Initial funding of this research was provided by crowd-funding donors who generously gave so that this project could get started—-thank you! Without these resources, this research would not have happened. 


\section{TABLE OF CONTENTS}

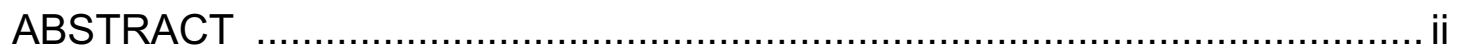

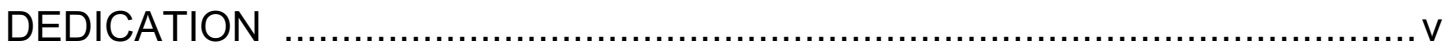

ACKNOWLEDGEMENTS …..........................................................

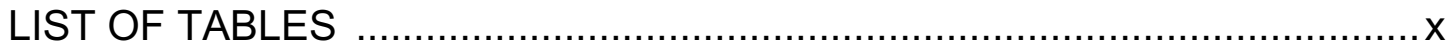

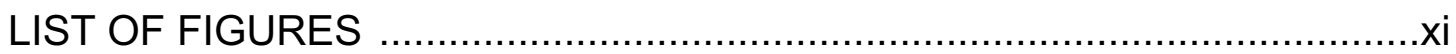

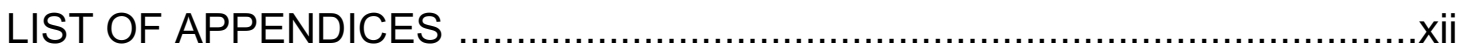

LIST OF ABBREVIATIONS …........................................................ii

CHAPTER

I. LITERATURE REVIEW

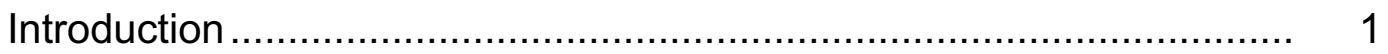

- Autism Background ......................................................... 1

- Diagnostic Criteria for Autism Spectrum Disorder .................... 1

- Using Neuroimaging to Investigate Sensory Processing Brain Mechanisms in Autism Spectrum Disorder...................... 3

- Focus of Dissertation and Research ...................................... 3

Definition and Importance Multisensory Integration ........................... 4

- Definition of Multisensory Integration ...................................... 4

- Importance of Multisensory Integration .................................... 7

- Using Neuroimaging to Investigate Underlying Sensory Processing Brain Mechanisms ................................................ 8

- A Brief Overview of Audio-visual Research in Autism Spectrum Disorder ........................................................... 10

- Natural versus Nonnatural Stimuli ................................ 11

- Static versus Dynamic Natural Stimuli .......................... 12

- Theories of Autism Spectrum Disorder Related to Sensory Processing ............................................... 14

Sensory Processing in Autism Spectrum Disorder ........................... 15

- Overview .................................................................... 15

- Definition of Complex Stimuli .................................................. 18

- Low-level Simple Processing in Autism Spectrum Disorder ...... 19

- Higher-order Complex Stimuli Processing in Autism

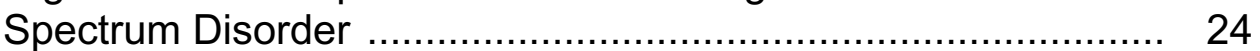

- Face Processing Complex Stimuli ......................................... 25

- Differentially Structurally Connected ........................................ 27

- Differentially Functionally Connected ……............................ 28

- The Role of Attention ........................................................... 29

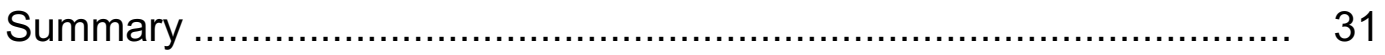

- Limitations and Future Directions ....................................... 33

- Gap in the Literature .................................................... 35

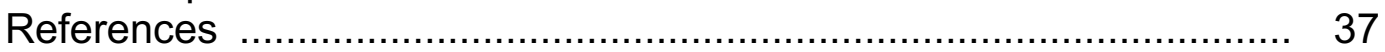


II. PROCESSING OF REAL-WORLD, DYNAMIC NATURAL STIMULI Page IN AUTISM IS LINKED TO CORTICOBASAL FUNCTION

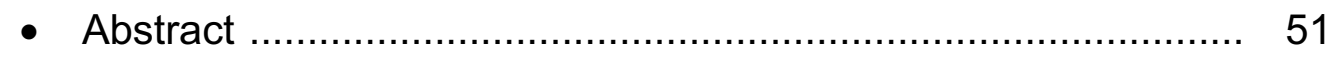

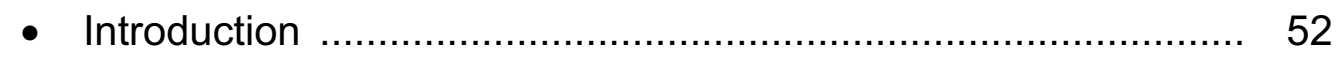

- Methods ....................................................................... 55

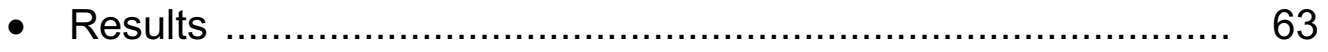

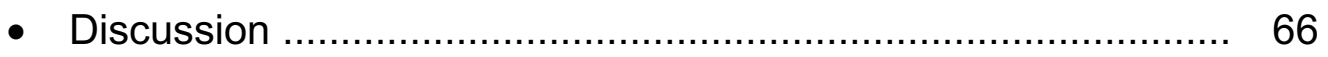

- References .................................................................. 72

- Tables ...................................................................... 81

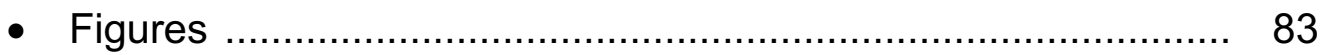

III. GENERAL DISCUSSION

- Brief Summary .................................................... 88

- Inferences for the Primary Results of this Study ................... 89

- Future Studies ............................................................... 91

- Final Conclusions .......................................................... 98

- References ............................................................ 99 


\section{LIST OF TABLES}

Table

Page

1. Group Demographic and Behavioral Data ................................... 81

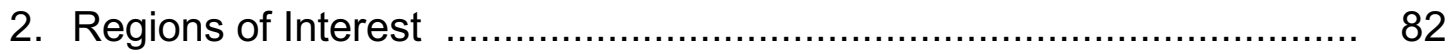




\section{LIST OF FIGURES}

Figure

Page

1. Functional Magnetic Resonance Imaging Task ................................ 83

2. Within-group whole-brain contrasts between conditions .................... 84

3. Between-group whole-brain contrasts .......................................... 86 


\section{LIST OF APPENDICES}

$\begin{array}{ll}\text { Appendix } & \text { Page }\end{array}$

1. Raven's Progressive Matrices ................................................... 101 


\section{LIST OF ABBREVIATIONS}

3D: three-dimensional

ADHD: attention deficit/hyperactivity disorder

ADOS: Autism Diagnostic Observation Schedule

AFNI: Analysis of Functional Neurolmages (software package)

ASD: autism spectrum disorder

AV: audio-visual (stimulus condition)

BG: basal ganglia

BOLD: blood-oxygen-level-dependent

DSM: Diagnostic and Statistical Manual

DTI: diffusion tensor imaging

EBA: extrastriate body area

EEG: electroencephalography

EP2D: echo planar pulse sequence

FBA: fusiform body area

fMRI: functional magnetic resonance imaging

FSIQ: full-scale intelligence quotient

FWHM: full-width half-max

GP: globus pallidus

IPS: intraparietal sulcus

IQ: intelligence quotient

MRI: magnetic resonance imaging

MPRAGE: magnetization-prepared rapid gradient-echo 
PDD: pervasive developmental disorder

PSC: percent signal change

$\mathrm{ROI}$ : region of interest

STG: superior temporal gyrus

TD: typically developed

TTL: transistor-transistor-logic

V: visual-only (stimulus condition)

WASI: Wechsler Abbreviated Scale of Intelligence 


\title{
CHAPTER I
}

\section{LITERATURE REVIEW}

\section{INTRODUCTION}

\author{
Autism Background
}

Autism Spectrum Disorder (ASD) is a neurodevelopmental disorder that produces life-long challenges in key areas including language and communication, social interactions, and responding to the environment. These challenges can significantly impact these individuals' ability to attend, learn, and interact with others. ASD affects 1 in 59 children in the United States (Baio et al., 2018), with a greater prevalence in males (1 in 38) than in females (1 in 152). It is widely accepted that genetics and environment combine to elicit various ASD phenotypes (J. A. Chen, Peñagarikano, Belgard, Swarup, \& Geschwind, 2015; Persico \& Bourgeron, 2006) contributing to the heterogeneity of this disorder with some individuals being more affected in certain aspects than others.

\section{Diagnostic Criteria for Autism Spectrum Disorder}

ASD is clinically diagnosed based on behavioral criteria as outlined in the Diagnostic and Statistical Manual-5 (DSM-5; American Psychiatric Association, 
2013). To receive an ASD diagnosis, symptoms must appear in the first few years of life and cannot be attributed to other disorders such as global developmental delay, intellectual developmental disorder, or other mental disorder. Clinically significant core deficit areas for an ASD diagnosis include; a) social communication and interactions, and b) restricted/repetitive behaviors or interests. The DSM-5 added sensory processing deficits as part of the clinical diagnostic criterion for the first time in ASD in 2013. Specifically, the DSM-5 identifies, "hyper- or hyporeactivity to sensory input or unusual interests in sensory aspects of the environment (e.g., apparent indifference to pain/temperature, adverse response to specific sounds or textures, excessive smelling or touching of objects, visual fascination with lights or movement)" with ASD. The DSM-5 criteria for sensory differences fall under the core deficit area of restricted, repetitive patterns of behavior, interests, or activities.

Considering the fact that more than $80 \%$ of individuals along the entire autism spectrum exhibit sensory processing and integration difficulties that are often seen early in childhood (Ben-Sasson et al., 2009), it is hypothesized that these sensory differences may contribute to other core deficits of ASD including attention, learning, social interactions, language development, anxiety, and repetitive behaviors (Bahrick \& Todd, 2012; Bednarz, Kana, Thye, Sartin, \& Herringshaw, 2017; Thye, Bednarz, Herringshaw, Sartin, \& Kana, 2016). Therefore, identifying and addressing the underlying neural mechanisms for sensory processing and integration deficits in ASD may help to elucidate the neural underpinnings of this disorder. Neuroimaging techniques provide a non-invasive 
way to characterize these neural mechanisms of behavioral sensory deficits in ASD.

Using Neuroimaging to Investigate Sensory Processing Brain Mechanisms in Autism Spectrum Disorder

Neuroimaging studies of sensory processing in ASD traditionally focused on auditory or visual processing, but more recent studies are investigating sensory integration, primarily audio-visual, since sensory inputs from one modality can influence how sensory information from another sensory modality is processed. Most of these studies have used artificial stimuli such as basic shapes and pure tones; however, these stimuli do not reflect the real-world. More recent studies have used real-world stimuli including static images of events or objects that we experience in our environment. However, only a few studies have included dynamic natural stimuli that most closely represent what we see and hear in our daily surroundings. To better understand the neural mechanisms that underly sensory processing and integration deficits in ASD that may contribute to higherorder cognitive deficits, studies are needed that utilize dynamic, real-world stimuli.

Focus of Dissertation and Research

This literature review focuses primarily on audio-visual multisensory integration in ASD with an emphasis on recent cross-modal neuroimaging studies with behavioral measures. Prevailing theories of autism proposed over the past 
twenty years related to functional and structural connectivity during multisensory integration in individuals with ASD will be reviewed specifically in light of the stimuli utilized (natural or nonnatural) and the corresponding differential task performance observed in individuals with autism. First, we begin by defining multisensory integration and its importance in sensory processing research in ASD.

\section{DEFINITION AND IMPORTANCE OF MULTISENSORY INTEGRATION}

\section{Definition of Multisensory Integration}

Multisensory integration is the integration of sensory inputs between multiple sensory modalities. Throughout the day, individuals are required to process a variety of incoming sensory inputs, filter out competing extraneous information, and integrate information from the various senses to form a unified precept of our surroundings (Powers, Hillock, \& Wallace, 2009). Inputs to one sensory modality can influence perception in another sensory modality when these inputs are temporally and spatially congruent (McDonald, Teder-Sälejärvi, \& Hillyard, 2000; Meredith, Nemitz, \& Stein, 1987). Audio-visual illusions are often used to test precise timing of audio-visual integration since they rely on precise timing of the information in order to perceive the illusion. One example of an audiovisual illusion commonly used to test audio-visual multisensory integration is the McGurk Effect (McGurk \& MacDonald, 1976). In this effect, participants are presented with mismatched visual and auditory information, which, if integrated, 
combines to elicit a different response. For example, if participants without autism see a human face vocalize "ba" but are presented with the sound "ga", they often ( $>90 \%$ of trials) indicate that the sound they perceived was "da". This audio-visual integration is observed in children and adults and relies on precise timing in the brain within and between cortical and subcortical sensory areas and is facilitated by multisensory neurons.

Neurons that elicit a response to multiple types of sensory stimuli are considered multisensory in comparison to unisensory neurons, which respond to inputs from only one sensory modality (Burnett, Stein, Perrault, \& Wallace, 2007; Meredith et al., 1987). While unimodal sensory pathways (auditory, visual, touch, olfactory, and taste) are known to process stimuli from a specific sensory modality (e.g., the visual pathway primarily processes visual inputs), stimuli from one sensory modality may also be processed to some degree by multiple sensory pathways via "multisensory neurons" (Sadato et al., 1996). For example, the superior colliculi are known to primarily process visual stimuli; however, multisensory neurons account for approximately $50-60 \%$ of the neurons in the superior colliculus. This has been demonstrated by lesioning the superior colliculus in cats, which decreased multisensory integration (auditory, visual, and somatosensory combinations) by $50 \%$ (Burnett et al., 2007). This reveals that even though the superior colliculi primarily process visual inputs, it also appears these subcortical structures are able to process information from other sensory modalities possibly to integrate the multiple inputs into a unified percept. 
Other brain regions have also been shown to integrate auditory and visual information including the temporal gyri (inferior, middle, and superior), superior temporal sulcus, middle frontal gyrus, inferior parietal lobe/temporal parietal junction, and thalamus (Beauchamp, Lee, Argall, \& Martin, 2004; Calvert, 2001; Ryan A. Stevenson, Kim, \& James, 2009). Measuring multisensory integration of auditory and visual inputs for speech stimuli in the superior temporal sulcus can be reliably done based on superadditivity (the response to the combined audiovisual stimuli is greater than the sum of the individual responses to auditory and visual inputs $[A V>A+V])$; however, superadditivity is not a good measure of sensory integration for all types of audio-visual stimuli or for all brain regions that integrate audio-visual stimuli (Calvert, 2001; Ryan A. Stevenson et al., 2014). While a response enhancement audio-visual integration may also be found in a subadditive way ( $\mathrm{AV}>\mathrm{A}$ and $\mathrm{AV}>\mathrm{V}$ and $\mathrm{AV}<\mathrm{A}+\mathrm{V})$, some audio-visual stimuli can result in a response depression $(\mathrm{AV}<\mathrm{A}$ and $\mathrm{AV}<\mathrm{V})$ (Ryan $\mathrm{A}$. Stevenson et al., 2014). This is largely dependent upon the method used to measure multisensory integration with recordings directly from cells often showing superadditive enhancements while neuroimaging methods sum or average responses to stimuli from many neurons, not all of which are multisensory.

Understanding the nuances of measuring multisensory integration has helped to refine methods for testing this ability in humans. This is crucial because the ability to integrate sensory inputs from multiple sensory modalities is vital to forming our perceptions of the environment and thus our understanding of the 
underlying neural mechanisms for crossmodal integration are key to better delineating where this ability goes awry in disorders such as autism.

Importance of Multisensory Integration

Multisensory integration is an experience-dependent capability of the brain. Early sensory experiences affect the postnatal development of normal neural circuitry and multisensory integration capabilities (Bengoetxea et al., 2012). Development of our ability to process and integrate sensory inputs begins as early as four months of age in typically developed children (Lewkowicz, 2002). Importantly, this time period coincides with an intense period of hyperplasia seen in most infants later diagnosed with ASD with excessive brain growth (head circumference exceeding 80th percentile) seen during the first few years after birth (Courchesne, Carper, \& Akshoomoff, 2003). The areas of hyperplasia are primarily in the regions of the brain that undergo the most development postnatally primarily the frontal lobe and to a lesser extent the temporal lobes. In addition, during this same developmental period, infants later diagnosed with ASD also show aberrant brain oscillations that are thought to alter experience-dependent plasticity (Gabard-Durnam et al., 2019). Because this early postnatal period is typically when infants experience significant synaptogenesis, myelination, and pruning, aberrant brain growth and activation seen during this time in infants later diagnosed with ASD are thought to impact functional and structural connectivity and underly core deficits seen in ASD including sensory processing. Neuroimaging 
studies investigating sensory processing differences seen in ASD can characterize the functional brain mechanisms that underly the behaviors seen in childhood used to diagnose this disorder.

Using Neuroimaging to Investigate Underlying Sensory Processing Brain Mechanisms

In order to depict underlying brain activity that contributes to behavioral phenotypes of ASD, neuroimaging studies have primarily utilized magnetic resonance imaging (MRI) and electroencephalography (EEG) techniques. EEG uses electrodes placed on the scalp to measure the summation of neural activity in the brain and has very good temporal resolution. Generally, brain activation measured by EEG in sensory processing research is in response to the presentation of a stimulus or multiple stimuli. However, EEG is not precise in localizing brain activation and thus has poor spatial resolution compared to MRI.

Task-based functional MRI (fMRI) is the primary neuroimaging technique used to investigate sensory processing and integration. While in the MRI scanner, participants are presented with stimuli and subsequent blood-oxygen-leveldependent (BOLD) measures are calculated based upon the hemodynamic response to the stimuli (Lindquist, Loh, Atlas, \& Wager, 2009). BOLD signals are based on relative levels of oxygenated to deoxygenated hemoglobin in the brain and are considered an indirect measure of neuronal activity as neurons with greater metabolic demands receive an increase in oxygenated blood after 
increased activity. BOLD activation levels are then measured at specific timepoints, coinciding with stimulus presentation, in each voxel in the brain, and can be group averaged, and then compared between or within groups. Because fMRI measures brain function in response to specific stimuli or in response to combinations of stimuli, this technology is well-suited to investigate brain function that contributes to sensory processing differences in ASD. BOLD levels at specific regions of interest in the brain for each person are also used to correlate brain activation levels with behavioral task data (e.g., reaction time) in order to explore brain-behavior relationships (Webster et al., 2017).

Both fMRI and EEG studies investigating sensory processing in ASD have been primarily focused on examining auditory and visual sensory modalities. This may be due in part to the fact that auditory and visual stimuli are more easily delivered to participants via headphones and computer displays as opposed to the delivery of smell or taste stimuli. Because auditory and visual sensory modalities are the primary senses engaged by most people to navigate the world, the integration of these two senses is a primary means by which we perceive things in our environment and thus the focus of this research. While EEG has been used to investigate brain activation in ASD for audio-visual sensory integration, the bulk of research in this area has been done using fMRI. Therefore, while some EEG studies of audio-visual integration in ASD are included in this literature review, the focus of this review is primarily on fMRI studies of auditory and visual sensory processing and integration in ASD. Next we will discuss the strengths of 
multisensory versus unisensory research and the use of natural versus nonnatural stimuli.

\section{A Brief Overview of Audio-visual Research in Autism Spectrum Disorder}

Prior neuroimaging research investigating sensory processing in ASD has primarily been unimodal with a greater emphasis on auditory and visual sensory modalities. However, input from one sensory modality is known to influence or alter how the brain processes and integrates information from other sensory modalities. For example, the addition of auditory input for a visual search task improves (reduces) the time it takes to locate the visual target (de Boer-Schellekens, Keetels, Eussen, \& Vroomen, 2013). Thus, investigating sensory processing in ASD from a multisensory integration perspective provides the greatest opportunity to delineate brain mechanisms in ASD for processing sensory inputs that better reflect the multisensory nature of the real world. Additionally, because the brain processes artificial (nonnatural) stimuli such as pure tones and basic shapes differently than it does natural stimuli (real-world images and sounds), the type of stimuli used to test audio-visual multisensory integration can have differential results seen in those with ASD (Ryan A Stevenson et al., 2018). Thus, the use of simple audio-visual stimuli may not reveal differences in multisensory integration of more complex natural stimuli. 
Natural versus Nonnatural Stimuli The bulk of auditory and visual unisensory studies investigating sensory processing in ASD have utilized artificial (nonnatural) stimuli such as pure tones and basic shapes. This is also true for the majority of more recent studies that have examined multisensory integration in ASD (Foss-Feig et al., 2010; Poole, Gowen, Warren, \& Poliakoff, 2017; Stewart et al., 2016). For example, Stewart et al., (2016) measured reaction time in response to unisensory and multisensory presentations of pure tones congruently presented with the appearance of a black dot on a computer monitor. Both typically developed (TD) children and adults and those with ASD showed improved reaction time in the multisensory condition compared to unisensory presentations of the auditory or visual stimuli. Because both groups showed multisensory facilitation (improved performance on a task when more than one stimulus is presented), this result was seen as an indication that individuals with ASD do not show impaired integration of simple, low-level sensory stimuli. However, while these stimuli are well-controlled and thus enable fine-grained investigations of responses to these low-level stimuli, such stimuli do not reflect the natural environment in which we live and interact. For example, a real photo of a house is a more complicated image than a drawing comprising shapes intended to represent a house (square on the bottom and triangle on top). Natural stimuli are generally considered to be more complex than corresponding nonnatural stimuli and require higher-order cognitive mechanisms for processing (Kana, Keller, Cherkassky, Minshew, \& Just, 2006; Magnée, de Gelder, van Engeland, \& Kemner, 2011; N. Russo, Mottron, Burack, \& Jemel, 2012; Sahyoun, Belliveau, Soulières, Schwartz, \& Mody, 2010; Ryan A 
Stevenson et al., 2018). Because the human brain processes nonnatural stimuli differently than it does natural stimuli, the use of nonnatural stimuli limits the generalizability of results of sensory processing differences in individuals with ASD.

Static versus Dynamic Natural Stimuli. More studies investigating sensory processing in ASD have begun to utilize natural stimuli that are more representative of the real world (Magnée et al., 2011; N. Russo et al., 2012; Sahyoun et al., 2010). However, the stimuli used in most of these studies are static and do not accurately reflect dynamic movement found in the real world. Most autism studies that have utilized dynamic, natural stimuli have been conducted using human faces paired with vocalizations (Megnin et al., 2012; Saalasti et al., 2012; Smith \& Bennetto, 2007; R.A Stevenson et al., 2014). However, face and language stimuli are unique since they are typically processed in very specific brain regions. The human face is able to be recognized primarily using the inferior occipital cortex and fusiform gyrus while other brain regions are involved in processing semantic and emotional aspects of the faces (Zhen, Fang, \& Liu, 2013). Functional language is processed in the human brain initially in the auditory system with higher-order processing involving Broca's and Wernicke's Areas as well as subcortical areas and networks between these regions (Chang, Raygor, \& Berger, 2015). Thus, results from audio-visual studies using face and language stimuli may not generalize well to investigations of audio-visual integration more broadly. 
Few studies have been conducted investigating audio-visual integration in ASD using dynamic, natural stimuli that did not contain facial or language stimuli (Noel, De Niear, Stevenson, Alais, \& Wallace, 2017; R.A Stevenson et al., 2014). Moreover, the videos used in these studies lasted for only a few seconds and then immediately after the participant was required to respond to a task and did not utilize neuroimaging to capture brain activation related to behavioral data. Only two studies investigating sensory processing in ASD (Bolton, Jochaut, Giraud, \& Van De Ville, 2018; Byrge, Dubois, Tyszka, Adolphs, \& Kennedy, 2015) used dynamic natural stimuli that lasted more than a few seconds, enabling the engagement of brain mechanisms that underly continuous processing of a dynamic, real-world scene with audio-visual content. Bolton et al., (2018) used a documentary video on sun exposure while Byrge et al., (2015) had participants watch an episode of a television show (The Office) while in the MRI. However, the videos used in these two studies were confounded with facial and/or language processing. In addition, the background in these dynamic videos changed throughout the videos, making interpretation of brain activation results more difficult with respect to the multitude of types of stimuli processed in any scene, which included faces, vocalizations, and biological motion. Thus, considering the lack of neuroimaging studies investigating audio-visual sensory processing in ASD using dynamic, natural stimuli, there is still a need to investigate multisensory integration in this disorder using stimuli that more closely represent the real world without confounds of facial or language processing. 
Various theories of sensory processing in ASD have been proposed to explain the results of studies that have used natural and nonnatural stimuli. What follows is a review of the predominant theories of ASD related to sensory processing and integration.

Theories of Autism Spectrum Disorder Related to Sensory Processing

Multiple theories have been put forward to explain the diverse phenotype of ASD since autism was first described in the 1940s (Kanner, 1943). Here we briefly discuss the prevailing theories related to sensory processing and integration in ASD.

Initially the Weak Central Coherence Theory (Frith \& Happé, 1994) sought to make sense of the differential performance (response time and accuracy) seen in individuals on the autism spectrum (Frith \& Happé, 1994). Individuals with ASD often perform better than typically developed peers without autism on tasks involving fine discriminations in research studies that utilized artificial stimuli such as pure tones and visual search tasks utilizing embedded figures (figures incorporated into complex shapes). Superior local, unimodal processing was proposed as a mechanism to explain this differential performance.

The Enhanced Perceptual Functioning Theory (Mottron, Dawson, Soulières, Hubert, \& Burack, 2006) later postulated a bias for superior local processing, but with an emphasis on lower-level operations that do not require topdown, higher-order cognitive processing. Around this same time the 
Underconnectivity Theory emerged in which long-distance interhemispheric and intrahemispheric (anterior-posterior) processing was proposed to be atypical in individuals with autism (Just, Cherkassky, Keller, \& Minshew, 2004). Theories have evolved and have been refined over the past decade or so, and now contain more fine-grained aspects such as analysis of temporal processing involved in sensory integration according to the Temporal Binding Window Hypothesis (Brock, Brown, Boucher, \& Rippon, 2002).

While each of these theories attempts to explain a specific facet of sensory processing in ASD, a universal theory has yet to be proposed that ties together the varied behavioral and neuroimaging results that reflect sensory differences in ASD. Next, these theories will be discussed in the context of the results of studies investigating audio-visual sensory processing in ASD.

\section{SENSORY PROCESSING IN AUTISM SPECTRUM DISORDER}

\section{Overview}

Sensory processing dysfunction is defined as "the inability to organize and use sensory information appropriately to make sense of the environment" (Ayres, 1972; Ayres \& Tickle, 1980). Atypical responses to non-noxious stimuli ranging from hypo-responsive to hyper-responsive characterize individuals with dysregulated sensory systems. Disorders that include sensory processing deficits include traumatic brain injury, anxiety disorders, mood disorders, dyslexia, and 
autism (Brout et al., 2018; Folmer, Billings, Diedesch-Rouse, Gallun, \& Lew, 2011; Hofmann \& Bitran, 2007; Vandermosten et al., 2010). While sensory processing dysfunction was not a diagnostic criterion for ASD in the DSM until 2013 (American Psychiatric Association, 2013), deficits in sensory processing have been wellcharacterized in most individuals along the entire autism spectrum since the time the disorder was first characterized (Kanner, 1943).

Sensory processing differences are a prevalent aspect of ASD (Marco, Hinkley, Hill, \& Nagarajan, 2011; Nieto, López, \& Gandía, 2017), which can make it difficult to participate in every-day activities (Ayres \& Tickle, 1980). For example, children with autism who are hypersensitive to lights may find it difficult to sit in a classroom with fluorescent lights that flicker, which can create a strobe light effect for some children. Similarly, noises ranging from a clock ticking to a fire alarm may negatively impact the ability of individuals with autism to attend or may overwhelm them and cause them to flee and seek a less sensory intensive space (Brout et al., 2018). Though commonly referred to as "self-stimulatory behaviors," we now know that some stereotypical repetitive behaviors such as hand-flapping and rocking may be a means by which many individuals with ASD attempt to self-regulate their sensory systems (Ashburner, Ziviani, \& Rodger, 2008; Minshew \& Hobson, 2008).

Making accommodations to help individuals with ASD regulate their sensory systems can decrease anxiety and have a positive impact on their ability to attend and learn (Howe \& Stagg, 2016; Pfeiffer, Duker, Murphy, \& Shui, 2019). Some examples of sensory interventions for children with ASD in the classroom include the use of seats with exercise balls that provide continuous vestibular and 
proprioceptive input, noise cancelling headphones, and sensory breaks as part of a sensory diet (Howe \& Stagg, 2016; Matin Sadr et al., 2017). While these interventions target the behavioral symptoms of sensory dysregulation, we need to better understand the underlying brain mechanisms that contribute to these sensory deficits.

Multisensory studies investigating audio-visual integration in ASD have had varied results, with some studies suggesting that those with ASD do not integrate sensory inputs similar to TD controls while other studies indicate they do integrate sensory information. Thus a primary question of multisensory integration research over the past decade or so has been to clarify whether or the extent to which individuals with ASD integrate sensory inputs and how differences in sensory integration might impact their performance on behavioral tasks. One factor contributing to the varied results is the type of stimuli used. Less complex auditory and visual stimuli such as pure tones and basic shapes that have no socially relevant aspects are often processed faster and with equivalent or greater accuracy by those with ASD compared to TD controls (Collignon et al., 2012; de Boer-Schellekens et al., 2013). Artificial stimuli may contain figures or abstract representations of things found in the real world or pure tones and white noise that are sounds not elicited from items found in the environment. Therefore, these are considered nonnatural stimuli. More complex stimuli include the human face, language, and static or dynamic images of things found in the real-world (e.g., animals; plants; tools) that can be semantically categorized. As stimuli become more complex and often more socially relevant, performance by many individuals 
with ASD can be negatively affected, impacting response times and accuracy (Bertone, Mottron, Jelenic, \& Faubert, 2005; Minshew \& Hobson, 2008). In order to discuss the differing results in ASD processing of low-level simple stimuli and complex stimuli, we next define what is meant by "complex".

\section{Definition of Complex Stimuli}

The complexity of a stimulus pertains to multiple aspects of the stimulus that can include (1) the level of detail in a figure or shape, (2) the amount of language content, (3) the amount of social information contained in the stimuli, and (4) the degree to which the stimulus represents real-world objects and events. While a basic shape such as a square would be considered a very simple stimulus, the arrangement of multiple shapes into a configuration would be considered a more complex stimulus. One example of a more complex figure is Raven's Progressive Matrices (Appendix 1), a test used to measure fluid intelligence (Raven, 2000). While its figures are considered to be more complex stimuli compared to basic shapes, individuals with ASD often show behavioral performance (e.g., reaction time) on the test's tasks that does not differ from controls (Yamada et al., 2012). Similarly, stimuli that contain letters are less complex than those that contain words or phrases. Stimuli containing language, especially human vocalizations, are considered socially relevant complex stimuli. In addition to spoken language, other

stimuli that are socially relevant contain information that would be important in a social context including the human face, images of real-world objects and events, 
and corresponding sounds that would naturally be elicited by those objects or events. Finally, static images of real-world objects or events are less complex compared to a dynamic version of that object or event. For example, a static photo of a person would be considered a complex, high-level stimulus, but it would be considered less complex from a cognitive processing perspective than a video of that person, especially if they are moving and/or speaking.

Thus stimuli that reflect the real-world, such as photos, are socially more complex than artificial representations of those stimuli (e.g., a drawing of a person) and dynamic real-world stimuli (e.g., a video of a person) are more complex than static images of the same person, object, scene, or event. Simpler stimuli make lower-level cognitive processing demands while more complex stimuli require higher-order cognitive processing. Next we discuss how individuals with ASD process low-level nonnatural stimuli and more complex (more socially relevant) natural stimuli that require higher-order processing.

\section{Low-level Simple Stimuli Processing in Autism Spectrum Disorder}

Results of studies assessing low-level sensory integration capabilities of individuals with ASD are varied. From a multisensory perspective, cues from one sensory modality can help facilitate processing of sensory inputs from another modality in typically developed individuals (Collignon et al., 2012). For example, an auditory pip (pure tone) is known to aid in a visual search for a colored bar oriented horizontally or vertically within a field of obliquely oriented colored bars (Van der Burg, Olivers, Bronkhorst, \& Theeuwes, 2008). This is known as the "pip- 
pop effect"-the auditory pip makes the visual target effectively "pop" out. In another study, an auditory cue (a $500 \mathrm{~Hz}$ tone) presented synchronously with a color change of the visual target (a horizontally oriented bar) in the search field was shown to improve accuracy and response time for typically developed children and adults (Collignon et al., 2012). However, the pip had no effect on the performance of ASD children and adults. Adolescents and adults with ASD generally demonstrated faster response times and greater accuracy than typically developed participants during the unimodal visual task (without the auditory cue). Behavioral performance of the typically developed participants began to reach that of ASD participants when the auditory cue (pip) was added. This result was seen as a lack of low-level audio-visual integration in individuals with ASD since their performance was not altered by the addition of sound stimuli, indicating that multisensory integration dysfunction in this population may be seen in processing simple stimuli and may not solely be applicable to processing more complex stimuli.

In contrast, the ability of individuals with ASD to integrate low-level sensory information was demonstrated reliably in an illusion that depends on the timing of the audio-visual inputs (Foss-Feig et al., 2010). Because precise timing of sensory inputs is critical to perceiving illusions, audio-visual illusions are often used to test multisensory integration. In typically developed individuals, perceiving audio-visual information as belonging to the same event relies on temporal binding of inputs from multiple sensory modalities. Illusions used in multisensory research test the boundaries of temporal binding windows as they leverage our innate ability to 
integrate inputs from various senses to perceive the illusion (Shams \& Kim, 2010; Williams, Massaro, Peel, Bosseler, \& Suddendorf, 2004). The "flash-beep" illusion takes advantage of auditory priming to elicit a visual illusion. When typically developed individuals hear two beeps (binaurally) synchronized with a single visual flash of light (a white circle on a black background), they perceive two flashes (circles) in their visual field; however, the further apart the auditory cue and visual stimuli are spaced in time (the more asynchronous), the less susceptible a person is to the illusion. This indicates that typically developed individuals have a "window of time" for exhibiting cross-sensory (audio-visual) interaction effects ( 150msec). To evaluate the timeframe within which ASD children integrate low-level audiovisual inputs, various stimulus onset asynchronies were utilized to identify a temporal window in which the flash-beep illusion could be elicited (Foss-Feig et al., 2010). They found that children and adolescents with ASD have a wider temporal binding window $(+/-300 \mathrm{msec})$ within which they perceive the flash-beep illusion. Therefore, the participants with ASD continued to perceive two flashes over an extended period of asynchrony of the audio-visual stimuli. Foss-Feig et al., (2010) surmised that individuals with ASD have the capability to integrate low-level sensory inputs (a pure tone and a white circle) but do so over a longer period of time.

These two studies using simple low-level audio-visual stimuli reached opposing conclusions about the ability of individuals with ASD to integrate such stimuli. One possible explanation for the different findings may be the age of the participants in the studies. While Foss-Feig et al., (2010) included children and 
adolescents, Collignon et al., (2012) included older adolescents and adults. Considering that multisensory integration ability can improve with age, it is possible that the inclusion of adults in the Collignon study was a factor in the lack of a group difference. The finding of a wider temporal binding window in individuals with ASD in the Foss-Feig study may account for earlier controversies in the literature surrounding differential performance on tasks utilizing simple stimuli.

This particular finding of an extended temporal binding window supports the Temporal Binding Deficit Hypothesis and has been confirmed by other studies (Kwakye, Foss-Feig, Cascio, Stone, \& Wallace, 2011; N. Russo et al., 2012). Thus, it appears that individuals with autism do integrate low-level sensory stimuli, but do so over an extended time period, differentially affecting their performance depending upon the task. One possible explanation for this altered temporal processing of low-level audio-visual stimuli is that it may have resulted from differential (global/local) connectivity during early development in children with ASD. Feasibly, more efficient systems are reinforced while less efficient, perhaps aberrantly connected networks were not strengthened as significantly during the early years of sensory processing development when hyperplasia is seen in children with ASD (Courchesne et al., 2003). Hypothetically, this may strengthen more efficient cortical networks at the expense of slower, less efficient networks resulting in differential higher-order/lower-level processing in children with ASD (Belmonte et al., 2004).

The differential performance seen in low-level sensory integration by individuals with ASD has also been attributed in part to their ability to focus to a 
greater degree on details of their environment at the expense of perceiving more holistic aspects. Theories of sensory processing based on this premise (Weak Central Coherence and Enhanced Perceptual Functioning) would conceivably indicate that superior performance on tasks involving detailed analysis would be facilitated by a brain system in which discrete pieces of information are processed more efficiently than more complex aspects of the same stimuli (Frith \& Happé, 1994; Mottron et al., 2006). A task that requires processing of low-level stimuli within a more complex image that typically involves global processing would help delineate aspects of global and local stimuli processing. Liu et al., (2011) used 3D line drawings in which the participants were asked to simply count the lines (essentially ignoring the three-dimensional [3D] aspects) and compared that to a task in which a judgment must be made as to whether the lined drawing is a $3 \mathrm{D}$ image. Processing 3D images relies on global processing (posterior visual and medial frontal [MF]) of the 3D aspects (Liu, Cherkassky, Minshew, \& Just, 2011). Processing discrete aspects of a complex image would theoretically be easier for an individual with autism based upon the above-mentioned theories as they often focus on details and neglect more holistic, universal aspects. While behavioral performance between the two groups was similar, differences were seen in brain activation patterns. Individuals with autism demonstrated the same cortical activation patterns regardless of the task, and thus were unaffected by irrelevant 3D spatial information when simply counting lines on a 3D image. However, typically developed individuals showed different activation between the two tasks - more MF and right superior frontal activation in the counting task that contained 
irrelevant 3D aspects. This demonstrated automatic global processing of the 3D imagery in the TD participants although it was not pertinent to the task. Thus, ASD participants did not experience interference of global processing networks and focused on the details of the image. These results are in line with the Weak Central Coherence and Enhanced Perceptual Functioning theories mentioned. Curiously, a comparison of functional connectivity (correlation of signal intensity between frontal and posterior visuospatial regions of interest) was similar between the two groups, which is in contrast to the Theory of Underconnectivity (Just et al., 2004). This may be due to differences in the types of stimuli used. The Theory of Underconnectivity and subsequent studies demonstrating underconnectivity utilized more complex stimuli such as sentence comprehension, while the stimuli used in Liu et al., (2011) would be considered less complex (simple shapes) requiring lower-level processing.

In summary, individuals with ASD do integrate low-level, simpler and less socially relevant sensory inputs, but often do so over a wider timeframe than TD individuals. Additionally, individuals with autism may behaviorally (reaction time and accuracy) perform at or above the level of TD participants on tasks involving analysis of details in low-level stimuli. This is in line with the phenotype of autism in which holistic-and perhaps more salient—aspects are not easily perceived. The inability to comprehend more general and global aspects of stimuli becomes more apparent as the stimuli become more complex and more socially relevant.

Higher-order Complex Stimuli Processing in Autism Spectrum Disorder 
Similar to studies investigating processing of simpler, low-level stimuli in individuals with autism, research examining ASD multisensory integration utilizing more complex, higher-order stimuli are almost exclusively focused on bimodal stimuli with an emphasis on audio-visual integration. In contrast to multisensory processing of lower-level stimuli such as tones and shapes, more aberrant processing of complex stimuli such as speech and environmental stimuli is often seen in individuals with autism. Since the majority of studies investigating audiovisual integration using complex stimuli have utilized human faces accompanied with vocalizations, we will first discuss results of studies using these unique realworld, dynamic stimuli.

Face Processing Complex Stimuli Individuals with autism often find it difficult to process facial features as people speak and to interpret facial expressions in a socially meaningful way. Some of the more complex visual stimuli used in ASD sensory processing research are human faces as they differ significantly from person to person and have many parts, some of which move as we speak. Facial processing involves specific areas of the brain (fusiform gyri; FG) and encompasses a unique field of ASD research that sets it apart from processing of less complex and very simple stimuli. It is believed that the specialization of the FG for facial processing develops during the first few years of life and is impacted greatly by our experience. Typically developed individuals utilize the FG for facial processing almost exclusively and engage the social brain network, which includes the FG and amygdala (Sato, Toichi, Uono, \& Kochiyama, 2012). Individuals with 
autism do not typically use the FG for processing facial expressions, but instead develop compensatory mechanisms for processing facial features including use of the primary visual cortex, fronto-central region and cerebellum (Joseph et al., 2016; Pierce, Müller, Ambrose, Allen, \& Courchesne, 2001). However, the areas activated vary significantly between ASD subjects. The heterogeneity of the autism disorder is reflected in the heterogeneity of the brain areas used by individuals with ASD to process facial expressions. Individuals with autism are known to process facial information in the same manner in which they process other forms of complex information - they visually focus on fine details and parts to the detriment of comprehending the face from a more holistic perspective. These results support theories that postulate superior local processing of stimuli requiring analysis of details at the detriment of processing more complex stimuli (Frith \& Happé, 1994).

The development of compensatory mechanisms by individuals with ASD to process faces is seen in multisensory integration of other types of complex stimuli. In a neuroimaging study examining processing differences between linguistic and visuospatial processing, children with ASD were presented with a pictorial problem-solving task in which they viewed visuospatial and semantic images and were required to complete the image series by selecting one of three options (Sahyoun et al., 2010). fMRI revealed differences in cortical activation between language-based problem-solving strategies utilized by typically developed children engaging the fronto-temporal brain regions and ASD participants, who utilized a more visuospatial strategy engaging occipito-temporal and ventral temporal regions. Thus, alternatively engaging differing brain regions appears to enable the 
participants with ASD to exhibit similar behavioral performance on the tasks (measured as response time and accuracy). This study also used Diffusion Tensor Imaging to characterize underlying structural connections pertinent to the regions of interests derived from the fMRI task. Decreased frontal connections (inferior frontal to middle temporal gyrus and fusiform gyrus) in children and adolescents with ASD supported the group differences found in the fMRI data for processing the visual semantic stimuli (e.g., a picture of a hammer), which showed that participants with ASD relied less on anterior processing of the stimuli than TD participants.

Other studies using diffusion tensor imaging (DTI) have found similar differential structural connectivity in ASD with only some networks being underconnected. Next we discuss findings of differential global and local connectivity in ASD.

\section{Differentially Structurally Connected}

Differentially connected (globally underconnected and locally overconnected) networks have been a focus of research in sensory integration processing in autism (Anderson, Druzgal, et al., 2011; Khan et al., 2013; Thomas, Humphreys, Jung, Minshew, \& Behrmann, 2010). Neuroimaging and behavioral studies have found conflicting evidence related to various aspects of differential connectivity. For example, a DTI study of white matter tracts in children with ASD showed decreased structural connectivity in all tracts investigated (uncinate 
fasciculus - bilaterally, corpus callosum, fornix, and superior longitudinal fasciculus) (Poustka et al., 2012). The most significant difference was seen in the uncinate fasciculus, which connects the inferior frontal and anterior temporal brain areas thought to be important in the regulation of emotional responses to auditory and visual inputs. This data supports a phenotype of autism in which emotional dysregulation is often a core deficit. While connectivity of the corpus callosum was decreased in comparison to typically developed individuals, it did not reach significance in Poustka et al., (2012). Because the corpus callosum is a core interhemispheric structure, this lack of a difference is contrary to the Theory of Underconnectivity proposed by Just et al. (2004) in which inferior performance during a sentence comprehension task was seen as a deficit in processing inputs that require global, anterior-posterior or interhemispheric coordination (Just et al., 2004). In a separate study, Just's lab found that the anterior portion of the corpus callosum, the genu, was significantly smaller in adults with ASD compared to typically developed participants (Kana et al., 2006). Identifying individuals with ASD based upon these types of structural and functional connectivity findings utilizing neuroimaging techniques has been done with $80 \%$ accuracy and may one day be utilized as one aspect to aid in early diagnosis (Anderson, Nielsen, et al., 2011). However, to date, there are no well-defined or universally accepted brain biomarkers for autism that are able to be utilized as diagnostic criteria for ASD.

\section{Differentially Functionally Connected}


Differential global and local functional connectivity (global underconnectivity and local overconnectivity) is also believed to be related to altered temporal processing of sensory stimuli. As addressed earlier, individuals with ASD are able to integrate sensory inputs from different sensory modalities, but they do so over an expanded period of time according to the Temporal Binding Deficit Hypothesis (Brock et al., 2002). This broader temporal binding window can alter performance on tasks depending upon the complexity of the stimuli (simple/low-level or complex/higher-order), with differential performance observed based upon the type of task and stimulus used (Foss-Feig et al., 2010; Hillock-Dunn \& Wallace, 2012). In addition, behavioral measures (response time and accuracy) of performance in individuals with ASD are typically at or above performance levels of typically developed participants depending upon the task. ASD individuals excel at certain tasks that utilize simple, low-level stimuli such as pure tones and shapes; however, group differences can vary between TD and ASD when more complex stimuli are tested. One explanation for these behavioral differences may be related to whether or not the study includes an attention task and how well a participant maintains attention/engagement during the task.

The Role of Attention

Attention has been shown to modulate multisensory integration performance in individuals on the autism spectrum (Mishra \& Gazzaley, 2012). In one study, adults with ASD viewed happy and fearful faces with congruent or 
incongruent happy or fearful voices (Magnée et al., 2011). When attention was divided between auditory and visual targets (auditory tone or white dot on the nose), typically developed individuals showed congruency effects as evidenced by increased amplitude in the N170 (negative event related potential [ERP] at 170ms post-stimulus) in the left occipital-temporal region when processing incongruent stimuli. Individuals with ASD did not display a similar congruency effect and thus the authors suggest that this demonstrates a lack of multisensory integration in the ASD group. However, when selective attention was manipulated by the presentation of numbers on the cheeks of the faces being viewed in some conditions, ASD individuals showed congruency effects similar to typically developed individuals. Thus manipulation of visual attention altered performance on the multisensory tasks in individuals with autism.

Other studies have investigated the use of simple stimuli (numbers on the face) during a selective attention task. As stated previously, individuals with autism focus on details of an image to the detriment of processing the image as a whole. Therefore, it would be expected that their performance in a selective attention task would be improved by the addition of simple stimuli such as numbers regardless of the complexity of the target stimuli (faces and voices). This is in line with the Weak Central Coherence and Enhanced Perceptual Functioning theories addressed earlier. For example, one study investigated whether typically developing infants could detect the amodal intersensory aspect of rhythm by alternating the rhythm of a hammer in a video (Bahrick \& Lickliter, 2000). They found that infants could discriminate when the information was presented 
bimodally (audio-visual), but not unimodally (visually or auditorily) as indicated by time spent looking at novel rhythm sequences reflecting their ability to selectively attend to a stimulus. Thus, selective attention is modulated by intersensory amodal features such as rhythm at an early age. Since multisensory integration is an experience-dependent capability and aberrant attention at an early age may impact experience-dependent abilities, deficits in selective attention may negatively impact a child's ability to develop multisensory integration.

In summary, the ability to process and integrate multiple sensory inputs is altered in individuals with autism based upon attentional demands. Though complex stimuli appear to be more challenging for individuals with autism to process and integrate, they demonstrate compensatory mechanisms that facilitate their behavioral performance (response time and accuracy) across various types of stimuli and tasks.

\section{SUMMARY}

Characterizing underlying brain mechanisms that contribute to behavioral aspects of ASD is critical to obtain a better understanding of the neural nature of this disorder. Individuals with ASD often exhibit atypical responses to sensory stimuli in one or more sensory modalities. Their responses can range from hyporesponsive to hyper-responsive, but how this altered responsivity develops is not entirely clear (Dunn \& Brown, 1997). Theories of sensory processing in ASD have focused on examining functional connectivity and temporal dynamics of sensory 
integration across sensory modalities. Neuroimaging studies exploring these aspects have utilized different stimuli and tasks to further delineate the mechanisms of processing both lower-level, simple stimuli and more complex, higher-order stimuli. Individuals with ASD often exhibit superior sensory integration on stimuli requiring lower-level processing, while increasingly complex stimuli more often result in differential processing schemes that can impact behavioral measures (including response time and accuracy) as well as comprehension of the information received (such as facial recognition). Compensatory strategies to process sensory inputs are evident in the differential temporal dynamics and use of different brain regions (often right lateralized or posterior) or visual strategies used by ASD individuals compared to those who are typically developed. Thus individuals with autism may develop compensatory mechanisms to process and integrate sensory information and do so more effectively with stimuli requiring bottom-up processing (early, stimulus driven) compared to top-down processing (later, cognitive perceptions) of more complex and often more socially relevant stimuli (Thye et al., 2016).

Development of compensatory processing mechanisms may be a factor differentiating higher-functioning individuals with autism from lower functioning individuals. Individuals on the autism spectrum who are more significantly affected by their autism may not have developed the compensatory mechanisms necessary to process every-day sensory experiences. Early diagnosis and early intervention may facilitate better outcomes for many children diagnosed with ASD. Neuroimaging techniques have been shown to be highly effective $(89 \%)$ in 
identifying children with autism based upon whole brain functional connectivity using functional MRI while at rest (Anderson, Nielsen, et al., 2011). Most children are diagnosed around the age of 4 years old though signs of autism can appear by the age of two as this is when behavioral differences more clearly begin to emerge compared to TD peers without autism. If neuroimaging can facilitate earlier diagnosis, children at risk could receive much-needed earlier interventions.

\section{Limitations of Neuroimaging in Autism Spectrum Disorder}

fMRI data collected from participants with ASD, who may be considered higher-functioning, may not generalize to other individuals with ASD who may find it difficult or impossible to attend for long periods of time, follow task instructions, lay very still for extended periods of time, and withstand the noisy and enclosed environment of an MRI. As a result, neuroimaging studies of sensory processing in this more limited sample of individuals with ASD are almost exclusively conducted with participants who are considered higher functioning from a cognitive and sensory perspective.

The term "high-functioning autism" is used in the literature to refer in general to individuals who are less affected by their autism. However, the use of this term can be confusing, since a person can be higher functioning (at or above typical levels of functioning) in one area and more affected in other areas. In addition, the use of this term is not well received by some in the ASD community as it implies that some individuals are thus "lower-functioning". Throughout this dissertation we 
use the term "high-functioning autism" to refer to individuals with ASD who are able to participate in neuroimaging studies in which they may need to follow task directions, hold very still for long periods of time, attend for extended time periods, and withstand the magnetic resonance imaging (MRI) scanner environment, which can be noisy and spatially restricted. We use this term because MRI studies are almost exclusively conducted with individuals with ASD who meet these criteria and thus are considered "higher-functioning" in relation to their ability to undergo task-based neuroimaging. A general limitation of MRI ASD research is the applicability of the results obtained to other individuals with ASD who are not able to perform such tasks in this sensory-intensive and spatially-restricted environment; therefore, researchers use the term "high-functioning" to avoid the implication that MRI research results are more broadly generalizable to all individuals with ASD. Whether or not an individual with ASD is able to undergo an MRI, they still meet the criteria for a diagnosis of ASD.

Consequently, it is not clear how conclusions drawn from this higherfunctioning subset of individuals apply to the broader group of individuals on the autism spectrum. Though autism is a heterogeneous disorder, individuals on the autism spectrum share common areas of deficit-social interaction, language/communication, repetitive behaviors, and restricted interests. It is possible that these common areas of deficit, though they are experienced to differing degrees across the spectrum, share common areas of neuronal dysfunction that will enable extrapolation of results to individuals more severely affected who are unable to participate in neuroimaging studies. 
Gaps in the Literature

Very few studies have utilized dynamic real-world stimuli to investigate sensory processing and integration in individuals who are typically developed as well as in individuals on the autism spectrum. While static images and short audio clips are well-controlled stimuli, they are not able to elicit brain responses that reflect the complexities of perceiving multisensory inputs in the environment. Therefore, more studies are needed that utilize various neuroimaging techniques to better understand how our senses work together to form our perception of the world and impact our response to information that we process.

Given the gap in the ASD literature with regard to multisensory integration of real-world dynamic information, we sought to address this by using fMRI to investigate audio-visual multisensory integration in ASD. Using this neuroimaging technique enables the characterization of brain mechanisms that underly behavioral phenotypes of sensory processing and integration differences seen in ASD. Therefore, the overarching goal of this dissertation research was to characterize differences in brain activation, using fMRI, in individuals with ASD compared to controls when processing real-world, dynamic audio-visual stimuli. To examine this, we created a five-minute dynamic video of a real-world scene (a person bouncing a ball) without any vocalization or facial processing confounds. Participants watched the video while in an MRI with a simple attention task to ensure participant engagement. We hypothesized that individuals with autism would show different levels of brain activation in primary auditory and visual cortical 
areas as well as in surrounding auditory and visual association cortical areas. We also hypothesized that cortical and subcortical brain regions where audio-visual information is known to be integrated (temporal parietal junction, pulvinar of the thalamus, and superior colliculus) would show differences between the groups. 


\section{REFERENCES}

American Psychiatric Association. (2013). Diagnostic and statistical manual of mental disorders (5td ed.).

Anderson, J. S., Druzgal, T. J., Froehlich, A., DuBray, M. B., Lange, N., Alexander, A. L., ... Lainhart, J. E. (2011). Decreased interhemispheric functional connectivity in autism. Cerebral Cortex (New York, N.Y. : 1991), 21(5), 1134-1146. https://doi.org/10.1093/cercor/bhq190

Anderson, J. S., Nielsen, J. a, Froehlich, A. L., DuBray, M. B., Druzgal, T. J., Cariello, A. N., ... Lainhart, J. E. (2011). Functional connectivity magnetic resonance imaging classification of autism. Brain : A Journal of Neurology, 134(Pt 12), 3742-3754. https://doi.org/10.1093/brain/awr263

Ashburner, J., Ziviani, J., \& Rodger, S. (2008). Sensory processing and classroom emotional, behavioral, and educational outcomes in children with autism spectrum disorder. American Journal of Occupational Therapy, 62(5), 564-573. https://doi.org/10.5014/ajot.62.5.564

Ayres, A. J. (1972). Sensory Integration and Learning Disorders. Western Psychological Services.

Ayres, A. J., \& Tickle, L. S. (1980). Hyper-responsivity to touch and vestibular stimuli as a predictor of positive response to sensory integration procedures by autistic children. American Journal of Occupational Therapy, 34, 375381. Retrieved from http://ajot.aota.org/

Bahrick, L. E., \& Lickliter, R. (2000). Intersensory Redundancy Guides Attentional 
Selectivity and Perceptual Learning in Infancy. Developmental Psychology, 36(2), 190-201. https://doi.org/10.1037//0012-1649.36.2.190.

Bahrick, L. E., \& Todd, J. T. (2012). Multisensory Processing in Autism Spectrum Disorders: Intersensory Processing Disturbance as a Basis for Atypical Development. In The new handbook of multisensory processes (pp. 657674). Cambridge, MA: MIT Press.

Baio, J., Wiggins, L., Christensen, D. L., Maenner, M. J., Daniels, J., Warren, Z., ... Dowling, N. F. (2018). Prevalence of autism spectrum disorder among children aged 8 Years - Autism and developmental disabilities monitoring network, 11 Sites, United States, 2014. MMWR Surveillance Summaries, 67(6). https://doi.org/10.15585/mmwr.ss6706a1

Beauchamp, M. S., Lee, K. E., Argall, B. D., \& Martin, A. (2004). Integration of Auditory and Visual Information about Objects in Superior Temporal Sulcus. Neuron, 41, 809-823. https://doi.org/10.1016/S0896-6273(04)00070-4

Bednarz, H. M., Kana, R. K., Thye, M. D., Sartin, E. B., \& Herringshaw, A. J. (2017). The impact of atypical sensory processing on social impairments in autism spectrum disorder. Developmental Cognitive Neuroscience, 29(April 2017), 151-167. https://doi.org/10.1016/j.den.2017.04.010

Belmonte, M. K., Allen, G., Beckel-Mitchener, A., Boulanger, L. M., Carper, R. a, \& Webb, S. J. (2004). Autism and abnormal development of brain connectivity. The Journal of Neuroscience, 24(42), 9228-9231. https://doi.org/10.1523/JNEUROSCI.3340-04.2004 Ben-Sasson, A., Hen, L., Fluss, R., Cermak, S. A., Engel-Yeger, B., \& Gal, E. 
(2009). A meta-analysis of sensory modulation symptoms in individuals with autism spectrum disorders. Journal of Autism and Developmental Disorders, 39(1), 1-11. https://doi.org/10.1007/s10803-008-0593-3

Bengoetxea, H., Ortuzar, N., Bulnes, S., Rico-Barrio, I., Lafuente, J. V., \& Argandoña, E. G. (2012). Enriched and deprived sensory experience induces structural changes and rewires connectivity during the postnatal development of the brain. Neural Plasticity, 2012, 305693.

https://doi.org/10.1155/2012/305693

Bertone, A., Mottron, L., Jelenic, P., \& Faubert, J. (2005). Enhanced and diminished visuo-spatial information processing in autism depends on stimulus complexity. Brain : A Journal of Neurology, 128(Pt 10), 2430-2441. https://doi.org/10.1093/brain/awh561

Bolton, T. A. W., Jochaut, D., Giraud, A. L., \& Van De Ville, D. (2018). Brain dynamics in ASD during movie-watching show idiosyncratic functional integration and segregation. Human Brain Mapping, 39(6), 2391-2404. https://doi.org/10.1002/hbm.24009

Brock, J., Brown, C. C., Boucher, J., \& Rippon, G. (2002). The temporal binding deficit hypothesis of autism. Development and Psychopathology, 14(2), 209224. Retrieved from http://www.ncbi.nlm.nih.gov/pubmed/12030688 Brout, J. J., Edelstein, M., Erfanian, M., Mannino, M., Miller, L. J., Rouw, R., ... Rosenthal, M. Z. (2018). Investigating misophonia: A review of the empirical literature, clinical implications, and a research agenda. Frontiers in Neuroscience, 12(February). https://doi.org/10.3389/fnins.2018.00036 
Burnett, L. R., Stein, B. E., Perrault, T. J., \& Wallace, M. T. (2007). Excitotoxic lesions of the superior colliculus preferentially impact multisensory neurons and multisensory integration. Experimental Brain Research, 179(2), 325338. https://doi.org/10.1007/s00221-006-0789-8

Byrge, L., Dubois, J., Tyszka, J. M., Adolphs, R., \& Kennedy, D. P. (2015). Idiosyncratic brain activation patterns are associated with poor social comprehension in autism. Journal of Neuroscience, 35(14), 5837-5850. https://doi.org/10.1523/JNEUROSCI.5182-14.2015

Calvert, G. A. (2001). Crossmodal Processing in the Human Brain: Insights from Functional Neuroimaging Studies. Cerebral Cortex, 11(12), 1110-1123. https://doi.org/10.1093/cercor/11.12.1110

Chang, E. F., Raygor, K. P., \& Berger, M. S. (2015). Contemporary model of language organization: an overview for neurosurgeons. Journal of Neurosurgeryl, 122(February), 250-261. https://doi.org/10.3171/2014.10.JNS132647.Disclosure Chen, J. A., Peñagarikano, O., Belgard, T. G., Swarup, V., \& Geschwind, D. H. (2015). The Emerging Picture of Autism Spectrum Disorder: Genetics and Pathology. Annual Review of Pathology: Mechanisms of Disease, 10(1), 111-144. https://doi.org/10.1146/annurev-pathol-012414-040405 Collignon, O., Charbonneau, G., Peters, F., Nassim, M., Lassonde, M., Lepore, F., ... Bertone, A. (2012). Reduced multisensory facilitation in persons with autism. Cortex; a Journal Devoted to the Study of the Nervous System and Behavior, 1-7. https://doi.org/10.1016/j.cortex.2012.06.001 
Courchesne, E., Carper, R., \& Akshoomoff, N. (2003). Evidence of brain overgrowth in the first year of life in autism. JAMA : The Journal of the American Medical Association, 290(3), 337-344. https://doi.org/10.1001/jama.290.3.337

de Boer-Schellekens, L., Keetels, M., Eussen, M., \& Vroomen, J. (2013). No evidence for impaired multisensory integration of low-level audiovisual stimuli in adolescents and young adults with autism spectrum disorders. Neuropsychologia, 51(14), 3004-3013. https://doi.org/10.1016/j.neuropsychologia.2013.10.005

Dunn, W., \& Brown, C. (1997). Factor Analysis on the Sensory Profile From. 51(1), 490-495.

Folmer, R. L., Billings, C. J., Diedesch-Rouse, A. C., Gallun, F. J., \& Lew, H. L. (2011). Electrophysiological assessments of cognition and sensory processing in TBI: applications for diagnosis, prognosis and rehabilitation. International Journal of Psychophysiology: Official Journal of the International Organization of Psychophysiology, 82(1), 4-15. https://doi.org/10.1016/j.ijpsycho.2011.03.005

Foss-Feig, J. H., Kwakye, L. D., Cascio, C. J., Burnette, C. P., Kadivar, H., Stone, W. L., \& Wallace, M. T. (2010). An extended multisensory temporal binding window in autism spectrum disorders. Experimental Brain Research, 203(2), 381-389. https://doi.org/10.1007/s00221-010-2240-4

Frith, U., \& Happé, F. (1994). Autism: beyond "theory of mind". Cognition, 50(13), 115-132. Retrieved from http://www.ncbi.nlm.nih.gov/pubmed/12212920 
Gabard-Durnam, L. J., Wilkinson, C., Kapur, K., Tager-Flusberg, H., Levin, A. R., \& Nelson, C. A. (2019). Longitudinal EEG power in the first postnatal year differentiates autism outcomes. Nature Communications, 10(1). https://doi.org/10.1038/s41467-019-12202-9

Hillock-Dunn, A., \& Wallace, M. T. (2012). Developmental changes in the multisensory temporal binding window persist into adolescence. Developmental Science, 15(5), 688-696. https://doi.org/10.1111/j.14677687.2012.01171.x

Hofmann, S. G., \& Bitran, S. (2007). Sensory-processing sensitivity in social anxiety disorder: relationship to harm avoidance and diagnostic subtypes. Journal of Anxiety Disorders, 21(7), 944-954. https://doi.org/10.1016/j.janxdis.2006.12.003 Howe, F. E. J., \& Stagg, S. D. (2016). How Sensory Experiences Affect Adolescents with an Autistic Spectrum Condition within the Classroom. Journal of Autism and Developmental Disorders, 46(5), 1656-1668. https://doi.org/10.1007/s10803-015-2693-1

Joseph, J. E., Zhu, X., Gundran, A., Davies, F., Clark, J. D., Ruble, L., ... Bhatt, R. S. (2016). Typical and atypical neurodevelopment for face specialization: An fMRI study. Journal of Autism and Developmental Disorders, 45(6), 1725-1741. https://doi.org/10.1586/14737175.2015.1028369.Focused Just, M. A., Cherkassky, V. L., Keller, T. a, \& Minshew, N. J. (2004). Cortical activation and synchronization during sentence comprehension in highfunctioning autism: evidence of underconnectivity. Brain : A Journal of 
Neurology, 127(Pt 8), 1811-1821. https://doi.org/10.1093/brain/awh199

Kana, R. K., Keller, T. a, Cherkassky, V. L., Minshew, N. J., \& Just, M. A. (2006). Sentence comprehension in autism: thinking in pictures with decreased functional connectivity. Brain : A Journal of Neurology, 129(Pt 9), 24842493. https://doi.org/10.1093/brain/awl164

Kanner, L. (1943). Autistic disturbances of affective contact (pp. 217-250). pp. 217-250.

Khan, S., Gramfort, A., Shetty, N. R., Kitzbichler, M. G., Ganesan, S., Moran, J. M., ... Kenet, T. (2013). Local and long-range functional connectivity is reduced in concert in autism spectrum disorders. Proceedings of the National Academy of Sciences of the United States of America. https://doi.org/10.1073/pnas.1214533110

Kwakye, L. D., Foss-Feig, J. H., Cascio, C. J., Stone, W. L., \& Wallace, M. T. (2011). Altered auditory and multisensory temporal processing in autism spectrum disorders. Frontiers in Integrative Neuroscience, 4(January 2011), 1-11. https://doi.org/10.3389/fnint.2010.00129

Lewkowicz, D. J. (2002). Heterogeneity and heterochrony in the development of intersensory perception. Cognitive Brain Research, 14(1), 41-63. Retrieved from http://www.ncbi.nlm.nih.gov/pubmed/12063129

Lindquist, M. A., Loh, J. M., Atlas, L. Y., \& Wager, T. D. (2009). Modeling the Hemodynamic Response Function in fMRI: Efficiency, Bias and Mismodeling. Neuroimage, 45(1), 187-198. https://doi.org/10.1038/jid.2014.371 Liu, Y., Cherkassky, V. L., Minshew, N. J., \& Just, M. A. (2011). Autonomy of 
lower-level perception from global processing in autism: evidence from brain activation and functional connectivity. Neuropsychologia, 49(7), 2105-2111. https://doi.org/10.1016/j.neuropsychologia.2011.04.005

Magnée, M. J. C. M., de Gelder, B., van Engeland, H., \& Kemner, C. (2011). Multisensory integration and attention in autism spectrum disorder: evidence from event-related potentials. PloS One, 6(8), e24196.

https://doi.org/10.1371/journal.pone.0024196

Marco, E. J., Hinkley, L. B. N., Hill, S. S., \& Nagarajan, S. S. (2011). Sensory processing in autism: a review of neurophysiologic findings. Pediatric Research, 69(5 Pt 2), 48R-54R. https://doi.org/10.1203/PDR.0b013e3182130c54

Matin Sadr, N., Haghgoo, H. A., Samadi, S. A., Rassafiani, M., Bakhshi, E., \& Hassanabadi, H. (2017). The impact of dynamic seating on classroom behavior of students with autism spectrum disorder. Iranian Journal of Child Neurology, 11(1), 29-36. https://doi.org/10.22037/ijcn.v11i1.11193

McDonald, J. J., Teder-Sälejärvi, W. A., \& Hillyard, S. A. (2000). Involuntary orienting to sound improves visual perception. Nature, 407(6806), 906-908. https://doi.org/10.1038/35038085

McGurk, H., \& MacDonald, J. (1976). Hearing Lips and Seeing Voices. Nature, $264,746-748$.

Megnin, O., Flitton, A., Jones, C. R. G., de Haan, M., Baldeweg, T., \& Charman, T. (2012). Audiovisual speech integration in autism spectrum disorders: ERP evidence for atypicalities in lexical-semantic processing. Autism Research : 
Official Journal of the International Society for Autism Research, 5(1), 3948. https://doi.org/10.1002/aur.231

Meredith, M. A., Nemitz, J. W., \& Stein, B. E. (1987). Determinants of Multisensory Integration in Superior Colliculus Neurons . I . Temporal Factors. Journal of Neuroscience, 7(10), 3215-3229.

Minshew, N. J., \& Hobson, J. a. (2008). Sensory sensitivities and performance on sensory perceptual tasks in high-functioning individuals with autism. Journal of Autism and Developmental Disorders, 38(8), 1485-1498.

https://doi.org/10.1007/s10803-007-0528-4

Mishra, J., \& Gazzaley, A. (2012). Attention distributed across sensory modalities enhances perceptual performance. The Journal of Neuroscience: The Official Journal of the Society for Neuroscience, 32(35), 12294-12302. https://doi.org/10.1523/JNEUROSCI.0867-12.2012

Mottron, L., Dawson, M., Soulières, I., Hubert, B., \& Burack, J. (2006). Enhanced perceptual functioning in autism: an update, and eight principles of autistic perception. Journal of Autism and Developmental Disorders, 36(1), 27-43. https://doi.org/10.1007/s10803-005-0040-7

Nieto, C., López, B., \& Gandía, H. (2017). Relationships between atypical sensory processing patterns, maladaptive behaviour and maternal stress in Spanish children with autism spectrum disorder. Journal of Intellectual Disability Research, 61(12), 1140-1150. https://doi.org/10.1111/jir.12435

Noel, J. P., De Niear, M. A., Stevenson, R., Alais, D., \& Wallace, M. T. (2017). Atypical rapid audio-visual temporal recalibration in autism spectrum 
disorders. Autism Research, 10(1), 121-129.

https://doi.org/10.1002/aur.1633

Persico, A. M., \& Bourgeron, T. (2006). Searching for ways out of the autism maze: genetic, epigenetic and environmental clues. Trends in Neurosciences, 29(7), 349-358. https://doi.org/10.1016/j.tins.2006.05.010

Pfeiffer, B., Duker, L. S., Murphy, A., \& Shui, C. (2019). Effectiveness of NoiseAttenuating Headphones on Physiological Responses for Children With Autism Spectrum Disorders. Frontiers in Integrative Neuroscience, 13(November), 1-14. https://doi.org/10.3389/fnint.2019.00065

Pierce, K., Müller, R. a, Ambrose, J., Allen, G., \& Courchesne, E. (2001). Face processing occurs outside the fusiform "face area" in autism: evidence from functional MRI. Brain : A Journal of Neurology, 124(Pt 10), 2059-2073.

Retrieved from http://www.ncbi.nlm.nih.gov/pubmed/11571222

Poole, D., Gowen, E., Warren, P. A., \& Poliakoff, E. (2017). Brief Report: Which Came First? Exploring Crossmodal Temporal Order Judgements and Their Relationship with Sensory Reactivity in Autism and Neurotypicals. Journal of Autism and Developmental Disorders, 47(1), 215-223.

https://doi.org/10.1007/s10803-016-2925-z

Poustka, L., Jennen-Steinmetz, C., Henze, R., Vomstein, K., Haffner, J., \& Sieltjes, B. (2012). Fronto-temporal disconnectivity and symptom severity in children with autism spectrum disorder. The World Journal of Biological Psychiatry: The Official Journal of the World Federation of Societies of Biological Psychiatry, 13(4), 269-280. 
https://doi.org/10.3109/15622975.2011.591824

Powers, A. R., Hillock, A. R., \& Wallace, M. T. (2009). Perceptual training narrows the temporal window of multisensory binding. The Journal of Neuroscience : The Official Journal of the Society for Neuroscience, 29(39), 12265-12274. https://doi.org/10.1523/JNEUROSCI.3501-09.2009

Raven, J. (2000). The Raven's Progressive Matrices: Change and Stability over Culture and Time. Cognitive Psychology, 41(1), 1-48.

https://doi.org/10.1006/cogp.1999.0735

Russo, N., Mottron, L., Burack, J. A., \& Jemel, B. (2012). Parameters of semantic multisensory integration depend on timing and modality order among people on the autism spectrum: evidence from event-related potentials. Neuropsychologia, 50(9), 2131-2141. https://doi.org/10.1016/j.neuropsychologia.2012.05.003

Saalasti, S., Kätsyri, J., Tiippana, K., Laine-Hernandez, M., von Wendt, L., \& Sams, M. (2012). Audiovisual speech perception and eye gaze behavior of adults with asperger syndrome. Journal of Autism and Developmental Disorders, 42(8), 1606-1615. https://doi.org/10.1007/s10803-011-1400-0

Sadato, N., Pascual-Leone, a, Grafman, J., Ibañez, V., Deiber, M. P., Dold, G., \& Hallett, M. (1996, April 11). Activation of the primary visual cortex by Braille reading in blind subjects. Nature, Vol. 380, pp. 526-528. https://doi.org/10.1038/380526a0

Sahyoun, C. P., Belliveau, J. W., Soulières, I., Schwartz, S., \& Mody, M. (2010). Neuroimaging of the functional and structural networks underlying 
visuospatial vs. linguistic reasoning in high-functioning autism.

Neuropsychologia, 48(1), 86-95.

https://doi.org/10.1016/j.neuropsychologia.2009.08.013

Sato, W., Toichi, M., Uono, S., \& Kochiyama, T. (2012). Impaired social brain network for processing dynamic facial expressions in autism spectrum disorders. BMC Neuroscience, 13(1), 99. https://doi.org/10.1186/1471-220213-99

Shams, L., \& Kim, R. (2010). Crossmodal influences on visual perception. Physics of Life Reviews, 7(3), 269-284. https://doi.org/10.1016/j.plrev.2010.04.006

Smith, E. G., \& Bennetto, L. (2007). Audiovisual speech integration and lipreading in autism. Journal of Child Psychology and Psychiatry, and Allied Disciplines, 48(8), 813-821. https://doi.org/10.1111/j.14697610.2007.01766.x

Stevenson, R.A, Siemann, J. K., Schneider, B. C., Eberly, H. E., Woynaroski, T. G., Camarata, S. M., \& Wallace, M. T. (2014). Multisensory Temporal Integration in Autism Spectrum Disorders. Journal of Neuroscience, 34(3), 691-697. https://doi.org/10.1523/JNEUROSCI.3615-13.2014

Stevenson, Ryan A., Ghose, D., Fister, J. K., Sarko, D. K., Altieri, N. A., Nidiffer, A. R., ... Wallace, M. T. (2014). Identifying and Quantifying Multisensory Integration: A Tutorial Review. Brain Topography, 27(6), 707-730. https://doi.org/10.1007/s10548-014-0365-7

Stevenson, Ryan A., Kim, S., \& James, T. W. (2009). An additive-factors design 
to disambiguate neuronal and areal convergence: Measuring multisensory interactions between audio, visual, and haptic sensory streams using fMRI. Experimental Brain Research, 198(2-3), 183-194.

https://doi.org/10.1007/s00221-009-1783-8

Stevenson, Ryan A, Segers, M., Ncube, B. L., Black, K. R., Bebko, J. M., Ferber, S., \& Barense, M. D. (2018). The cascading influence of multisensory processing on speech perception in autism. Autism, 22(5), 609-624. https://doi.org/10.1177/1362361317704413

Stewart, C. R., Sanchez, S. S., Grenesko, E. L., Brown, C. M., Chen, C. P., Keehn, B., ... Müller, R. A. (2016). Sensory Symptoms and Processing of Nonverbal Auditory and Visual Stimuli in Children with Autism Spectrum Disorder. Journal of Autism and Developmental Disorders, 46(5), 15901601. https://doi.org/10.1007/s10803-015-2367-z

Thomas, C., Humphreys, K., Jung, K.-J., Minshew, N., \& Behrmann, M. (2010). The anatomy of the callosal and visual-association pathways in highfunctioning autism: a DTI tractography study. Cortex; a Journal Devoted to the Study of the Nervous System and Behavior, 47(7), 863-873. https://doi.org/10.1016/j.cortex.2010.07.006

Thye, M. D., Bednarz, H. M., Herringshaw, A. J., Sartin, E. B., \& Kana, R. K. (2016). The impact of atypical sensory processing on social impairments in autism spectrum disorder. Developmental Cognitive Neuroscience, (May), 0-1. https://doi.org/10.1016/j.den.2017.04.010

Van der Burg, E., Olivers, C. N. L., Bronkhorst, A. W., \& Theeuwes, J. (2008). 
Pip and pop: nonspatial auditory signals improve spatial visual search. Journal of Experimental Psychology. Human Perception and Performance, 34(5), 1053-1065. https://doi.org/10.1037/0096-1523.34.5.1053

Vandermosten, M., Boets, B., Luts, H., Poelmans, H., Golestani, N., Wouters, J., \& Ghesquière, P. (2010). Adults with dyslexia are impaired in categorizing speech and nonspeech sounds on the basis of temporal cues. Proceedings of the National Academy of Sciences of the United States of America, 107(23), 10389-10394. https://doi.org/10.1073/pnas.0912858107

Webster, P. J., Skipper-Kallal, L. M., Frum, C. A., Still, H. N., Ward, B. D., \& Lewis, J. W. (2017). Divergent Human Cortical Regions for Processing Distinct Acoustic-Semantic Categories of Natural Sounds: Animal Action Sounds vs. Vocalizations. Frontiers in Neuroscience, 10(January), 1-18. https://doi.org/10.3389/fnins.2016.00579

Williams, J. H. G., Massaro, D. W., Peel, N. J., Bosseler, A., \& Suddendorf, T. (2004). Visual-auditory integration during speech imitation in autism. Research in Developmental Disabilities, 25(6), 559-575. https://doi.org/10.1016/j.ridd.2004.01.008

Yamada, T., Ohta, H., Watanabe, H., Kanai, C., Tani, M., Ohno, T., ... Hashimoto, R. (2012). Functional alterations in neural substrates of geometric reasoning in adults with high-functioning autism. PLOS ONE, 7(8). https://doi.org/10.1371/journal.pone.0043220

Zhen, Z., Fang, H., \& Liu, J. (2013). The Hierarchical Brain Network for Face Recognition. PLoS ONE, 8(3). https://doi.org/10.1371/journal.pone.0059886 


\title{
CHAPTER II
}

PROCESSING OF REAL-WORLD, DYNAMIC NATURAL STIMULI IN AUTISM

IS

\section{LINKED TO CORTICOBASAL FUNCTION}

(this article has been accepted for publication in Autism Research)

Paula J Webster ${ }^{1}$, Chris Frum ${ }^{1}$, Amy Kurowski-Burt ${ }^{2}$, Christopher E. Bauer ${ }^{1}$, Sijin Wen $^{3}$, Jad H. Ramadan ${ }^{1}$, Kathryn A. Baker ${ }^{1}$, James W. Lewis ${ }^{1}$

${ }^{1}$ Rockefeller Neuroscience Institute, Department of Neuroscience, West Virginia University. ${ }^{2}$ Department of Human Performance, Division of Occupational Therapy, West Virginia University. ${ }^{3}$ Department of Biostatistics, West Virginia University.

\begin{abstract}
Many individuals with autism spectrum disorder (ASD) have been shown to perceive every-day sensory information differently compared to peers without autism. Research examining these sensory differences has primarily utilized nonnatural stimuli or natural stimuli using static photos with few having utilized dynamic, real-world non-verbal stimuli. Therefore, in this study we used functional magnetic resonance imaging to characterize brain activation of individuals with
\end{abstract}


high-functioning autism when viewing and listening to a video of a real-world scene (a person bouncing a ball) and anticipating the bounce. We investigated both multisensory and unisensory processing and hypothesized that individuals with ASD would show differential activation in (a) primary auditory and visual sensory cortical and association areas, and in (b) cortical and subcortical regions where auditory and visual information is integrated (e.g., temporal parietal junction, pulvinar, superior colliculus). Contrary to our hypotheses, the whole-brain analysis revealed similar activation between the groups in these brain regions. However, compared to TD controls the ASD group showed significant hypoactivation in left intraparietal sulcus (IPS) and left putamen/globus pallidus (GP). We theorize that this hypoactivation reflected underconnectivity for mediating spatio-temporal processing of the visual biological motion stimuli with the task demands of anticipating the timing of the bounce event. The paradigm thus may have tapped into a specific left-lateralized aberrant corticobasal circuit or loop involved in initiating or inhibiting motor responses. This was consistent with a dual "when versus where" psychophysical model of corticobasal function, which may reflect core differences in sensory processing of real-world, non-verbal natural stimuli in ASD.

\section{INTRODUCTION}

The ability to integrate sensory inputs from our environment enables us to make sense of the world and underlies the development of basic skills in children 
such as language acquisition and motor coordination (Kwakye et al., 2011; Lewkowicz \& Ghazanfar, 2009). Atypical sensory processing and integration is thought to contribute to core deficits in ASD including social interactions, language development, and repetitive behaviors (Bahrick \& Todd, 2012; Bednarz et al., 2017; Thye et al., 2016). The integration of auditory and visual inputs requires coordination between the primary cortical sensory regions and sensory association cortices as well as with subcortical areas that integrate auditory and visual information (N. Russo et al., 2012; Stein, Stanford, \& Rowland, 2009). Prior research has demonstrated that individuals with ASD differ from controls when integrating different modalities of sensory inputs for non-natural stimuli such as basic shapes and pure tones (de Boer-Schellekens et al., 2013; Foss-Feig et al., 2010) as well as complex natural stimuli such as speech (Megnin et al., 2012; Ryan A. Stevenson et al., 2017; Thye et al., 2016) or static real-world images (N. Russo et al., 2012). However, these temporal differences can vary depending on the complexity of the stimuli (Feldman et al., 2018; Noel et al., 2017; Ryan A. Stevenson \& Wallace, 2013). Some studies have investigated multisensory processing in ASD using real-world, dynamic stimuli. For instance, studies using dynamic stimuli have varied in the duration of stimuli presentation and task demands, including short video clips of a few seconds followed immediately by a forced-alternative choice task selection (R.A Stevenson et al., 2014), live images followed by a forced-alternative choice task (Greenfield, Ropar, Smith, Carey, \& Newport, 2015), or passive movie watching with no task (Gabrielsen et al., 2018; Natalie Russo et al., 2010). Only two studies (Bolton et al., 2018; Byrge et al., 
2015) have used the same video for all participants that was a continuous, dynamic video of a real-world scene that also included auditory information; however, the movies required relatively higher order processing of faces and verbal information. Thus, many gaps remain with regard to the nature and degree of stimulus complexity and/or task complexity that may potentially unmask more specific processing differences.

Because task performance of individuals with ASD has been shown to vary depending upon the task and stimuli complexity, the primary goal of this study was to investigate cortical and subcortical differences in brain activation between individuals with ASD and typically developed (TD) peers when viewing and listening to a precisely controlled real-world stimulus that would not be confounded by face or language processing. To achieve this we created a continuous video of a real-world scene (person bouncing a basketball) with two conditions (audiovisual and visual-only) to investigate group differences in processing the same stimuli from both a multisensory and unisensory perspective, while participants underwent MRI scanning with a simple attention task requiring anticipating when the ball would hit the floor.

In addition to examining group differences in brain activity, the resulting fMRI data were used to explore relationships between brain activation and behavior related to sensory processing and ASD severity. Our first hypothesis was that compared to controls, the ASD group would show differences in brain activation in primary auditory cortices (Heschl's Gyrus), primary visual cortices (V1), and surrounding associated auditory and visual cortical areas, and that these 
differences would correlate with behavioral assessment measures of sensory processing. Our second hypothesis was that individuals with ASD would differ from controls in activation levels in brain regions known to integrate auditory and visual information (Csonka, Mardmomen, Webster, Frum, \& Lewis, In Review; Lewis, 2010) including cortical areas (superior temporal gyrus [STG] and temporal parietal junction [TPJ]), and subcortical areas (pulvinar and superior colliculus [SC]). Identifying any such differences would advance a more detailed understanding of how sensory and task information is processed between individuals with versus without autism.

\section{METHODS}

\section{Participants}

Adolescent and adult participants were recruited from the surrounding region (Table 1 ) including a typically developed (TD) control group $(n=35)$ and an ASD group $(n=27)$. Inclusion criteria for all participants entailed initial screening for medical conditions that would exclude them from safely being able to undergo an MRI, were between the ages of 11-29 years old, native English speakers, had selfreported normal hearing and vision or wore corrective lenses, and had a Full-scale Intelligence Quotient (FSIQ-4) above 70 based on the Wechsler Abbreviated Scale of Intelligence (WASI). The ASD group had self-reported existing clinical diagnoses of Autistic Disorder, Asperger Syndrome, or Pervasive Developmental Disorder-not otherwise specified. ASD diagnoses were confirmed using the Autism 
Diagnostic Observation Schedule (ADOS-2) conducted by a research-reliable ADOS administrator (Lord et al., 2000). ADOS-2 Module 3 was used with three adolescents (age 12, 13, and 14 years old) and Module 4 was used for adults and adolescents ( $n=17$ age 13-27 years old) based upon verbal fluency. TD participants had no ASD diagnosis, no first-degree relatives with ASD, and no psychiatric disorders. Exclusion criteria listed here are followed by the number of participants subsequently excluded from each group for each criterion in parentheses: history of head trauma ( $n=2$ TD), psychiatric disorders including ADHD, anxiety, or depression ( $n=6$ TD), medical comorbidities beyond general ASD population ( $n=2$ ASD), family history of ASD ( $n=1$ TD), visual deficits that were not corrected with MRI compatible lenses ( $n=1$ ASD), non-native English speaker ( $n=1$ TD), fell asleep several times in the MRI ( $n=2$ TD; $n=1$ ASD), and undiagnosed ASD later suspected ( $n=1$ TD). Additional technical exclusion criteria included excess head motion ( $>15 \%$ of data was removed; $n=1$ TD), fMRI task errors (omissions) that exceeded the outlier limit ( $n=1$ ASD), technical issues with MRI data retrieval ( $n=1$ ASD), and no bilateral auditory activation when contrasting between the conditions (audio-visual minus visual-only; $n=1$ ASD). The final groups included $n=21$ TD and $n=20$ ASD participants retained who were matched for age (two-tailed $t$-test $p=0.31$ ), gender (Pearson Chi-Square $p=0.80$ ), handedness (Pearson Chi-Square $p=0.14$ ), and non-verbal IQ (Perceptual Reasoning Composite Score; two-tailed $t$-test $p=0.56)$. Informed consent was obtained from adult participants and from the parent/legal guardian of participants under 18 years of age as well as assent from adolescent participants based on 
procedures approved by the West Virginia University Institutional Review Board. Participants were paid for their time.

\section{Behavioral Testing}

Intelligence Quotient (IQ) Full Scale, Verbal, and Perceptual Reasoning IQ were measured using the Wechsler Abbreviated Scale of Intelligence (Wechsler, 2011). Participants were matched for Perceptual Reasoning IQ (Table 1 ; two-tailed $t$-test $p=0.56$ ) as these scores reflect non-verbal and visuomotor spatial reasoning skills and thus were more germane to the MRI non-verbal task used in this study, providing the most appropriate method for matching ASD participants with controls given the common language deficits in ASD (Mottron, 2004). Mean IQ scores were all in the average to above average range, though there was a significant difference in Full-scale IQ (FSIQ-4; two-tailed $t$-test $p=0.037)$. IQ testing was done under the supervision of a licensed Clinical Psychologist.

Sensory Profile Behavioral measures of sensory processing were based on the Adolescent/Adult Sensory Profile (Brown \& Dunn, 2002) and scored by an Occupational Therapist experienced in administration and scoring who was blinded to the participants' group. This self-report questionnaire uses a 5-point Likert Scale to assess behavioral responses to sensory inputs in six sensory domains (Auditory, Visual, Vestibular, Touch, Multisensory, Taste/Smell) each containing questions that reflect sensory behaviors in four quadrants (Low 
Registration, Sensation Seeking, Sensory Sensitivity, and Sensation Avoiding) with higher scores indicating greater severity.

Task Performance Participant task performance ("press the button when you perceive/think the ball hits the floor') while in the MRI was monitored throughout the video runs via a receiver in the control room with a light that indicated a button press had been made.

\section{Design and Stimuli}

We conducted a $2 \times 2$ (condition $\times$ group) block design study. The audio-visual stimulus (Figure 1) consisted of a dynamic (continuous) video acquired with a highdefinition video camera and edited using commercially available software (QuickTime Player, version 10.3). The actor in the video intermittently bounced a basketball on an auditorium stage. He was visually cued when to dribble the ball and was filmed from the neck down to avoid confounding facial processing (Pierce et al., 2001). Task blocks were either audio-visual (AV) or visual-only (V). Note that an audio-only condition with this stimulus paradigm would not be feasible as the absence of sound prior to a bounce does not provide any predictive sensory input for performing the task of anticipating the ball bounce and otherwise would effectively become a reaction time task. The video lasted 302 seconds with 14 task blocks ( 10 sec each) in each run ( $7 \mathrm{AV}$ and $7 \mathrm{~V}$ ) with either 8 or 9 bounces in each 10 sec task block totaling 119 bounces per run. Task blocks were pseudorandomly distributed between 15 non-task (rest) blocks (10 sec each) during which the actor stood still holding the ball. Each presentation of the video began with the 
words "GET READY" presented for 6 seconds, which faded into the first non-task block, providing an additional $6 \mathrm{sec}$ for steady state MRI acquisition. Each run ended with the words "KEEP HOLDING STILL" to remind participants they should not move even though the video was done. Two runs were created in which the task blocks were counter-balanced between the two runs. Each run was presented twice for a total of 4 runs (Run $A, B, A, B$ ).

fMRI Task While in the 3-Tesla Siemens Verio MRI, participants wore ear buds (Model S14, Sensimetrics Corp., Malden, MA) to hear the audio. A sound check prior to data acquisition assured that the participant could hear the sound in both ears over the continuous scanner noise. Participants were instructed to "press the button when you perceive/think the ball hits the floor" with their left index finger via a button box. The attention task was implemented to ensure participants were attending to the video and was designed to be very simple in order to decrease any confounds of brain activity that may be generated from more complex attentional tasks (Magnée et al., 2011). Instructions for the basketball-bouncing task were reviewed while the participant was in the scanner. A test run verified that the participant understood the instructions, that the sound levels were appropriate, and that the audio could be heard over the scanner noise. Sound intensity levels for the video were measured after the MRI scanning session with no significant difference found between the groups (sound pressure level [dB] ASD Mean=94.1 \pm 9.4 ; TD Mean=89.2 \pm 6.2 ; two-tailed $t$-test $p>0.10$ ). Participants viewed the video via a mirror mounted on the MRI head coil as the video was back-projected onto a 30-inch MRI compatible monitor subtending $8^{\circ}$ of angle vertically. The video was 
presented via a Windows PC computer with Presentation software (Neurobehavioral Systems). Researchers could see and hear the video from the control room and button presses were continuously monitored throughout via a receiver in the MRI control room. Participant performance was used to calculate task errors (omissions and commissions).

MRI Data Acquisition MRI data were acquired on a Siemens 3-Tesla Verio MRI scanner using a 12-channel head coil, which had ample space to allow participants wearing corrective lenses to fit comfortably. A T1-weighted MPRAGE pulse sequence $\left(1.5 \mathrm{~mm}\right.$ sagittal slices, $0.625 \times 0.625 \mathrm{~mm}^{2}$ in-plane resolution, $\mathrm{Tl}=2300 \mathrm{~ms}$ ) was used to acquire anatomical images while participants watched a movie of their choice. While in the MRI participants watched a task-based video (Figure 1). Blood Oxygen-Level Dependent (BOLD) signals were continuously collected using an echo planar pulse sequence $(\mathrm{ep} 2 \mathrm{~d}$; TR=2000ms, TE $=30 \mathrm{~ms}$, Field of View $=240 \mathrm{~mm}, 75^{\circ}$ flip angle). Continuous acquisition was used to best model the hemodynamic response to the real-world, dynamic stimuli. Brain volumes were collected in the axial plane with $4 \mathrm{~mm}$ slice thickness across $30-35$ slices (to include the entire cerebellum for all participants), and in-plane resolution of $3.75 \times 3.75$ with $4.6 \mathrm{~mm}$ thickness. The video was triggered by the $3 \mathrm{rd}$ MRI TTL pulse providing an additional six seconds for steady-state acquisition prior to the start of the video.

MRI Data Processing MRI data were processed using Analysis of Functional Neurolmages (AFNI) software (Cox, 1996). A non-linear affine transformation of anatomical data to MNI space (@SSwarper) using the MNI152 template was first 
performed, the ouput of which was used for alignment of echo planar imaging (EPI) data using standard AFNI procedures (afni_proc.py) including accounting for steady-state acquisition, despiking, temporal alignment, identification of motion outliers $(>3 \mathrm{~mm}), 6 \mathrm{~mm}$ FWHM blur, resampling of voxels to $3.5 \mathrm{~mm}^{3}$, and normalization of BOLD values to mean percent signal change (PSC) (Taylor et al., 2018). In AFNI, the time series BOLD was scaled to the voxel-wise mean using the BOLD signal across that run (Taylor et al., 2018). A per-subject regression analysis used motion parameters per run as regressors of no interest, a $2^{\text {nd }}$ order polynomial modeled any linear drift in the signal, and general linear trends were based on timing files for the two conditions (AV versus V). At the individual level, the output of afni_proc.py was evaluated. Canonical BOLD activation $(A \vee>V)$ in bilateral superior temporal gyrus (STG) for $\mathrm{AV}$ minus $\mathrm{V}$, along with right motor cortex activation (left-hand button press) for each task condition minus Mean $=0$ confirmed the functional data were processed correctly at the individual level. Alignment of EPI functional data output from afni_proc.py with the MNI152 template was also verified at the individual subject level.

Region of Interest (ROI) Derivation At the group level, a brain mask was created (3dmask_tool) from the combined individual subject masks (mask_epi_anat), which constituted the intersection of each participants EPI and anatomical data. The group mask was used in subsequent group analyses in AFNI to restrict the volume within which AFNI analyzes BOLD data (G. Chen, Saad, Nath, Beauchamp, \& Cox, 2012). The beta-coefficients for each subject for each condition were included in the AFNI multivariate ANOVA model (3dMVM) with age 
as a covariate. Main and interaction effects for group and condition were also included in the 3dMVM analysis in AFNI. The output of the 3dMVM ANOVA was thresheld $(t=3.56 p=0.001)$ in the AFNI viewer to compare BOLD PSC values at each voxel for between-group contrasts for each condition $\left(A S D_{A v}\right.$ minus $T D_{A V}$; $A S D \vee$ minus $T D \vee)$ and within-group contrasts between the conditions $\left(A_{S} D_{A V}\right.$ minus $A S D_{V}$ and $T D_{A v}$ minus $T D_{V}$ ). Resulting regions of interest (ROls) (Table 2) were cluster corrected (3dClustSim; $\alpha<0.05, P=0.001$ ).

BOLD PSC Extraction ROI masks were created from the brain volumes of those ROls that survived thresholding in the AFNI viewer and subsequent cluster correction. The ROI masks were then used to extract BOLD PSC values (3dRO/stats) for each subject for each condition and used for further statistical analysis (correlations with behavioral data, generate histograms of group averaged BOLD data, and post-hoc power analyses) in SPSS (version 26.0).

Correlations Correlational analyses were conducted in SPSS (version 26.0) between BOLD PSC values for each ROI and 1) task commission errors for each condition ( $\mathrm{V} V$ and $\mathrm{V}$ ) and 2) Adolescent/Adult Sensory Profile Quadrant Scores for both groups, as well as 3) ADOS-2 calibrated severity scores for the ASD group only. Correction for multiple comparisons at false detection rate (FDR) $q=0.05$ (Benjamini \& Hochberg, 1995).

\section{Statistical Analyses}

A multivariate analysis was performed in AFNI on the group averaged BOLD PSC values using $3 d M V M$. The output of that analysis was thresheld in AFNI 
$(t=3.56 p=0.001)$ and cluster corrected $(\alpha<0.05, p=0.001)$ to identify the brain regions that differed significantly from the between-group and within-group analyses. The resulting $\mathrm{ROI}$ volumes were used to generate $\mathrm{ROI}$ masks to extract the BOLD data for each subject and condition. F-tests of the extracted BOLD PSC data were conducted in the statistical package $R$ (version 3.6.0) to investigate main and interaction effects for group, condition, and task performance (commissions). Data normality was tested using the Shapiro-Wilk test in SPSS (version 26.0) with non-normally distributed data analyzed using the Kruskal-Wallis test and normally distributed data analyzed using the appropriate $t$-test (unpaired $t$-tests for betweengroup comparisons and paired $t$-tests for within-group comparisons). In SPSS, Levene's test for significance of variance was used to test homogeneity of variances. Pearson's Chi-Squared test was used for analysis of group differences of nominal data in SPSS. BOLD PSC values in all ROls were analyzed in SPSS for correlations with task commissions and Sensory Profile Quadrant Scores for both groups and with Autism Calibrated Severity Scores for the ASD group only. The Benjamini-Hochberg rank procedure was used to correct for multiple comparisons of correlation testing. Power analyses were conducted using G*Power ANOVA post hoc F-test. Tests were significant at $p<0.05$.

\section{RESULTS}

\section{Behavioral}


Sensory Profile Quadrant Scores As expected, the ASD group had higher (more severe) scores than the TD group on behavioral aspects of sensory processing (Table 1; two-tailed $t$-tests) including Low Registration $(p<0.001)$ and Sensation Avoiding $(p=0.02)$. However, the ASD group had lower scores for Sensation Seeking compared to controls (two-tailed $t$-test $p=0.001$ ).

Task Performance The task data was not normally distributed with a high number of commissions in the ASD group (Table 1). These commissions occurred primarily at the end of the task blocks after the ball had stopped bouncing and were seen in a subset $(n=6)$ of ASD participants. The groups did not differ in total task errors (omissions and commissions; $p=0.15$ ); however, there was a significant group difference in total commissions for both conditions ( $\mathrm{AV}$ commissions $p=0.02$; $V$ commissions $p=0.04)$. The commission error data was further examined in the statistical package $\mathrm{R}$ for possible main and interaction effects.

\section{fMRI}

Within-groups whole-brain contrasts $\left(A S D_{A v}\right.$ minus $A S D_{v}$; $T D_{A v}$ minus $\left.T D_{V}\right)$ showed significantly greater fMRI BOLD activation in the audio-visual condition compared to the visual-only condition in bilateral superior temporal gyrus (STG) for both groups (Figure 2, Table 2). The groups showed similar BOLD PSC activation in all STG ROls. Analysis of variance revealed a significant main effect of condition in all STG ROIs (TD left STG $\left[F_{1,74}=56.62, p<0.0001\right.$, Cohen $F=0.657$, TD right STG $\left[F_{1,74}=74.63, p<0.0001\right.$, Cohen $\left.F=0.76\right]$, ASD left STG $\left[F_{1,74}=55.88\right.$, 
$p<0.0001$, Cohen $F=0.64]$, ASD right STG $\left[F_{1,74}=83.64, p<0.0001\right.$, Cohen $\left.F=0.81\right]$.

Power analyses revealed power $\geq .99$ for all main effects of condition in all STG ROIs. No significant main effect for group or task commissions was found and there was no significant interaction in the STG ROIs. The TD group contrast also revealed an AV > V ROI that spanned the bilateral pulvinar and superior colliculus. In the BOLD PSC data extracted from this ROI, the groups showed similar levels of activation (TD $p<0.001, d=1.08$; ASD $p=0.01, d=0.49$ ) with a main effect for condition $\left(F_{1,74}=11.99, p=0.0009\right.$, Cohen $F=0.358$, Power=0.93).

Between-groups whole-brain analysis conducted in AFNI for each condition $\left(\mathrm{ASD}_{\mathrm{AV}}\right.$ minus $\mathrm{TD}_{\mathrm{AV}} ; \mathrm{ASD}_{\mathrm{V}}$ minus $\mathrm{TD} \vee$ ) revealed significant group differences in the visual-only contrast $(\mathrm{ROI}$ derivation $t=3.56, p=0.001$, cluster corrected at $\alpha<0.05, p=0.001$ ) in left putamen/GP and left IPS (Figure 3, Table 2). BOLD values extracted for these ROls showed significant hypoactivation in the ASD group compared to the TD group for both conditions (Figure 3 histogram). There was a significant main effect of group in the left IPS ROI $\left[F_{1,74}=41.96, p<0.0001\right.$, Cohen $F=0.61$, Power=0.99], a main effect of group in the left putamen/GP ROI $\left[F_{1,74}=37.04, p<0.0001\right.$, Cohen $F=0.430$, Power $\left.=0.97\right]$, a main effect of task performance (commissions) in the left putamen/GP ROI $\left[F_{1,74}=9.03, p<0.004\right.$, Cohen $F=0.19$, Power $=0.84$ ], and a group $x$ commission interaction effect in the left putamen/GP $\left(F_{1,74}=7.94, p=0.006\right.$, Cohen $F=0.186$, Power=0.79). The wholebrain ANOVA for the audio-visual contrast $\left(\mathrm{ASD}_{\mathrm{AV}}\right.$ minus $\left.\mathrm{TD}_{\mathrm{AV}}\right)$ did not result in 
any ROls that withstood thresholding in AFNI $(t=3.56 p=0.001)$ and subsequent cluster correction $(\alpha<0.05 p=0.001)$.

Relationships Between Brain Activation and Behavior While there was a main effect for commissions for the putamen/GP ROI, no correlations were found that survived correction for multiple comparisons between BOLD PSC and commission task errors, ADOS calibrated severity scores, or Sensory Profile Quadrant scores.

\section{DISCUSSION}

This study investigated how brain activity during processing of a dynamic realworld scene differed between adolescents and young adults with high-functioning ASD and their peers without ASD (a video of a person bouncing a basketball), examining brain activity from both a multisensory and unisensory perspective. The secondary goal of this study was to explore possible relationships between functional neural differences and behavioral measures of sensory processing and ASD severity. We hypothesized that the ASD group would differ from controls in primary and association auditory and visual sensory cortical areas and in cortical and subcortical regions where audio-visual information is known to be integrated, such as the TPJ, pulvinar, and SC. However, the data did not support these sensory-related hypotheses. Rather, the results revealed other differences between the groups with significant hypoactivation in the left IPS and left 
putamen/GP for the ASD group compared to controls. Theories related to underconnectivity and motor disinhibition mediated by corticobasal loops are proposed as underlying mechanisms for the neuroimaging findings as addressed below.

As expected, within-group contrasts ( $A S D_{A V}-A S D_{V}$; $\left.T D_{A V}-T D_{V}\right)$ showed that both groups demonstrated similar activation (AV > V) in bilateral STG, including primary auditory and association cortices, demonstrating that our task manipulation worked (Figure 2). These findings suggest that when processing this real-world, dynamic stimulus, high-functioning individuals with ASD were similar to controls in auditory and visual sensory cortical brain areas, as well as in subcortical brain regions (pulvinar and SC) generally reported to integrate auditory and visual information. The lack of a group difference in extracted BOLD values in the present study was not inconsistent with a recent meta-analysis in that audio-visual integration differences between those with ASD and controls was greater when using linguistic stimuli than with non-linguistic stimuli (Feldman et al., 2018), which was explicitly avoided in the present study.

In the between-group contrasts $\left(A S D_{A V}-T D_{A V} ; A S D_{V}-T D_{V}\right)$, again contrary to our hypothesis, the groups did not show any differences in primary auditory or visual cortical areas or in subcortical areas for integrating auditory and visual information. However, the ASD group showed significant hypoactivation in the left putamen/GP and left IPS compared to controls (Figure 3). Regarding this somewhat surprising finding, the location of the left IPS ROI was in line with parietal ROIs reported in other studies investigating audio-visual integration in ASD (Stickel 
et al., 2019). The IPS is also reported to receive sensory input from other cortical areas, including visual information from the extrastriate body area (EBA), which is a primary region in the brain for processing human biological motion (Downing, Jiang, Shuman, \& Kanwisher, 2001; Weiner \& Grill-Spector, 2011). A recent electroencephalography (EEG) study in children and adolescents with autism (Kröger et al., 2014) found reduced activation for processing biological motion stimuli. However, the lack of a group difference in the EBA, and also the fusiform body area (FBA) (Amoruso, Couto, \& Ibáñez, 2011; Schwarzlose, Baker, \& Kanwisher, 2005) may indicate that the groups in the present study were using this brain region similarly and thus the hypoactivation in the IPS may be better explained by reduced connectivity in the ASD group between the left EBA and left IPS. This theory is supported by an fMRI study that found adults with ASD were underconnected between the EBA and parietal lobe (McKay et al., 2012).

The hypoactivation in the left IPS in the ASD group may also have been contributing to the relative hypoactivation in the left putamen/GP. Based on a recent connectomic study, the IPS was reported to project visuomotor information directly to the putamen (Cacciola et al., 2017) and thus a corticobasal circuit may be impacting activation levels in this IPS-putamen/GP circuit. In addition, the IPS also projects to the frontal eye field (FEF) as part of the dorsal attention network (Vossel, Geng, \& Fink, 2014), and thus hypoactivation in the left IPS may impact downstream input to the left putamen/GP via multiple pathways.

The left-lateralized hypoactivation seen in the ASD group in the present study was consistent with two resting state functional connectivity (rsfMRI) studies that 
reported atypical right-hemisphere lateralization of motor circuit connectivity in ASD (Cardinale, Shih, Fishman, Ford, \& Muller, 2013; Floris et al., 2016). Consequently, performing the task of anticipating when the basketball would bounce based on visual motion information may have been readily processed by left corticobasal circuits in the TD group. The task was simple enough that the ASD group could perform at the same level of accuracy as measured by omission button press task errors. However, the ASD group showed an effect of commission errors, suggesting that they may in fact have been struggling with disinhibition mediated by the corticobasal circuits needed to stop pressing the button when the ball stopped bouncing since most of the commission errors occurred at the end of the task blocks. This interpretation was supported by the results showing six ASD participants who consistently made commission errors in the form of adding one last button press at the conclusion of most blocks (AV or $\mathrm{V}$ ). Given this interpretation, whether the hypoactivated corticobasal circuit revealed in the present study reflected (1) spatio-temporal attention demands related to sensory event anticipation, (2) the mechanics of preparing and executing a left-hand button press motor response (Unruh et al., 2019), or (3) signaling when to stop performing a repetitive task remains a question for future studies. Regardless, these results were consistent with an earlier study of response timing deficits in autism suggesting atypical left hemisphere striatal chronometric systems (D'Cruz et al., 2009), as well as dual "when versus where" theory of corticobasal function with regard to motor control, involving the putamen versus caudate nucleus, respectively (Findlay \& Walker, 1999; Watanabe \& Munoz, 2011). In particular, the 
spatial location of the ball bounce in the present study was highly predictable, while anticipating the timing of the bounce required more effort that may have taxed this IPS-putamen loop differently.

These findings should be considered in light of limitations related to this study. Our sample size for the ASD group was relatively small considering the heterogeneous nature of those with ASD; therefore, these results may not generalize across the broader spectrum of the ASD population. Additionally, adults and adolescents with ASD may not show similar differences compared to controls (Noel, De Niear, Van Der Burg, \& Wallace, 2016; Nomi \& Uddin, 2015), thus, age may represent another behavioral dimension to consider. In hindsight, capturing eye-tracking data during fMRI scanning would have benefited data interpretation especially as it relates to the differences seen in brain regions involved in visuomotor interactions and the relationship with commission errors in the ASD group. Lastly, considering the high rate of commission errors in the ASD group and the putamen ROI group difference, data analysis would have benefited from correlations with ASD repetitive behavior scores (Abbott et al., 2018) that are well characterized in evaluation tools such as the Repetitive Behavior Scale and the Repetitive Behavior Questionnaire.

In summary, contrary to our main hypotheses, we did not find any group differences in brain activation in auditory or visual sensory or association cortical areas nor in brain regions involved in audio-visual sensory integration per se. However, group differences were found in a left-lateralized corticobasal circuit involving the IPS and putamen/GP. The underconnectivity theory of autism (Just, 
Keller, Malave, Kana, \& Varma, 2012) would predict local overconnectivity and long-distance underconnectivity in the ASD group. The results of the present study support the underconnectivity theory if IPS hypoactivation is related to interhemispheric underconnectivity (left/right IPS) or intrahemispheric underconnectivity (left EBA to IPS, or left IPS to putamen/GP). Moreover, they newly support a dual "when versus where" theory of corticobasal function with regard to movement initiation.

Acknowledgments

We greatly appreciate the time and dedication of the participants and their families without whom this research would not have been possible. Also, we would like to acknowledge the resource contributions of Vanderbilt University, West Liberty University, Marshall University, West Virginia University (WVU) Rockefeller Neuroscience Institute, and the WVU Carruth Center. For valuable contributions regarding design and analysis, we thank Dr. Jodi Lindsey, Dr. Marc Haut, Dr. Shuo Wang, and Dr. Runnan Cao. Funding was provided by a grant from the West Virginia Clinical and Translational Science Institute (NIH/NIGMS Award Number 5U54GM104942-03), and crowd-funding donors. Funding entities were not involved in the study design or data collection and analysis. 


\section{REFERENCES}

Abbott, A. E., Linke, A. C., Nair, A., Jahedi, A., Alba, L. A., Keown, C. L., Fishman, I., Müller, R. A. (2018). Repetitive behaviors in autism are linked to imbalance of corticostriatal connectivity: A functional connectivity MRI study. Social Cognitive and Affective Neuroscience, 13(1), 32-42. https://doi.org/10.1093/scan/nsx129

Amoruso, L., Couto, B., \& Ibáñez, A. (2011). Beyond extrastriate body area (EBA) and fusiform body area (FBA): Context integration in the meaning of actions. Frontiers in Human Neuroscience, 5(November), 1-3. https://doi.org/10.3389/fnhum.2011.00124

Bahrick, L. E., \& Todd, J. T. (2012). Multisensory Processing in Autism Spectrum Disorders: Intersensory Processing Disturbance as a Basis for Atypical Development. In The new handbook of multisensory processes (pp. 657674). Cambridge, MA: MIT Press.

Bednarz, H. M., Kana, R. K., Thye, M. D., Sartin, E. B., \& Herringshaw, A. J. (2017). The impact of atypical sensory processing on social impairments in autism spectrum disorder. Developmental Cognitive Neuroscience, 29(April 2017), 151-167. https://doi.org/10.1016/j.den.2017.04.010

Benjamini, Y., \& Hochberg, Y. (1995). Controlling the False Discovery Rate : A Practical and Powerful Approach to Multiple Testing. Journal of the Royal Statistical Society, 57(1), 289-300.

Bolton, T. A. W., Jochaut, D., Giraud, A. L., \& Van De Ville, D. (2018). Brain 
dynamics in ASD during movie-watching show idiosyncratic functional integration and segregation. Human Brain Mapping, 39(6), 2391-2404. https://doi.org/10.1002/hbm.24009

Brown, C., \& Dunn, W. (2002). Adolescent/Adult Sensory Profile manual. San Antonia, TX: Psychological Corporation.

Byrge, L., Dubois, J., Tyszka, J. M., Adolphs, R., \& Kennedy, D. P. (2015). Idiosyncratic brain activation patterns are associated with poor social comprehension in autism. Journal of Neuroscience, 35(14), 5837-5850. https://doi.org/10.1523/JNEUROSCI.5182-14.2015

Cacciola, A., Calamuneri, A., Milardi, D., Mormina, E., Chillemi, G., Marino, S., Naro, A., Rizzo,G., Anastasi, G., Quartarone, A. (2017). A connectomic analysis of the human basal ganglia network. Frontiers in Neuroanatomy, 11(September). https://doi.org/10.3389/fnana.2017.00085

Cardinale, R. C., Shih, P., Fishman, I., Ford, L. M., \& Muller, R.-A. (2013). Pervasive rightward asymmetry shifts of functional networks in autism spectrum disorders. JAMA Psychiatry, 70(9), 975-982.

https://doi.org/10.1001/jamapsychiatry.2013.382

Chen, G., Saad, Z. S., Nath, A. R., Beauchamp, M. S., \& Cox, R. W. (2012). FMRI group analysis combining effect estimates and their variances. Neurolmage, 60(1), 747-765. https://doi.org/10.1016/j.neuroimage.2011.12.060

Cox, R. W. (1996). AFNI: Software for Analysis and Visualization of Functional Magnetic Resonance Neuroimages. Computers and Biomedical Research, 
29(3), 162-173. https://doi.org/10.1006/cbmr.1996.0014

Csonka, M., Mardmomen, N., Webster, P., Frum, C., \& Lewis, J. W. (n.d.). A meta-analysis of brain networks involved in audio-visual interactions at a category level. submitted.

D’Cruz, A. M., Mosconi, M. W., Steele, S., Rubin, L. H., Luna, B., Minshew, N., \& Sweeney, J. A. (2009). Lateralized Response Timing Deficits in Autism. Biological Psychiatry, 66(4), 393-397.

https://doi.org/10.1016/j.biopsych.2009.01.008

de Boer-Schellekens, L., Keetels, M., Eussen, M., \& Vroomen, J. (2013). No evidence for impaired multisensory integration of low-level audiovisual stimuli in adolescents and young adults with autism spectrum disorders. Neuropsychologia, 51(14), 3004-3013.

https://doi.org/10.1016/j.neuropsychologia.2013.10.005

Downing, P. E., Jiang, Y., Shuman, M., \& Kanwisher, N. (2001). A cortical area specialized for visual processing of the human body. Journal of Vision, 1(3), 2470-2474. https://doi.org/10.1167/1.3.341

Feldman, J. I., Dunham, K., Cassidy, M., Wallace, M. T., Liu, Y., \& Woynaroski, T. G. (2018). Audiovisual multisensory integration in individuals with autism spectrum disorder: A systematic review and meta-analysis. Neuroscience and Biobehavioral Reviews, 95(September), 220-234.

https://doi.org/10.1016/j.neubiorev.2018.09.020

Findlay, J. M., \& Walker, R. (1999). A model of saccade generation based on parallel processing and competitive inhibition. Behavioral and Brain 
Sciences, 22(4), 661-674. https://doi.org/10.1017/S0140525X99002150

Floris, D. L., Barber, A. D., Nebel, M. B., Martinelli, M., Lai, M. C., Crocetti, D., Baron-Cohen, S., Suckling, J., Pekar, J.J., Mostofsky, S. H. (2016). Atypical lateralization of motor circuit functional connectivity in children with autism is associated with motor deficits. Molecular Autism, 7(1).

https://doi.org/10.1186/s13229-016-0096-6

Foss-Feig, J. H., Kwakye, L. D., Cascio, C. J., Burnette, C. P., Kadivar, H., Stone, W. L., \& Wallace, M. T. (2010). An extended multisensory temporal binding window in autism spectrum disorders. Experimental Brain Research, 203(2), 381-389. https://doi.org/10.1007/s00221-010-2240-4

Gabrielsen, T. P., Anderson, J. S., Stephenson, K. G., Beck, J., King, J. B., Kellems, R., Top, D.N., Russell, N.C.C., Anderberg, E., Lundwall, R.A., Hansen, B., South, M. (2018). Functional MRI connectivity of children with autism and low verbal and cognitive performance. Molecular Autism, 9(1), 114. https://doi.org/10.1186/s13229-018-0248-y

Greenfield, K., Ropar, D., Smith, A. D., Carey, M., \& Newport, R. (2015). Visuotactile integration in autism: atypical temporal binding may underlie greater reliance on proprioceptive information. Molecular Autism, 6(51).

https://doi.org/10.1186/s13229-015-0045-9

Just, M. A., Keller, T. a, Malave, V. L., Kana, R. K., \& Varma, S. (2012). Autism as a neural systems disorder: a theory of frontal-posterior underconnectivity. Neuroscience and Biobehavioral Reviews, 36(4), 1292-1313. https://doi.org/10.1016/j.neubiorev.2012.02.007 
Kröger, A., Bletsch, A., Krick, C., Siniatchkin, M., Jarczok, T. A., Freitag, C. M., \& Bender, S. (2014). Visual event-related potentials to biological motion stimuli in autism spectrum disorders. Social Cognitive and Affective Neuroscience, 9(8), 1214-1222. https://doi.org/10.1093/scan/nst103

Kwakye, L. D., Foss-Feig, J. H., Cascio, C. J., Stone, W. L., \& Wallace, M. T. (2011). Altered auditory and multisensory temporal processing in autism spectrum disorders. Frontiers in Integrative Neuroscience, 4(January 2011), 1-11. https://doi.org/10.3389/fnint.2010.00129

Lewis, J. W. (2010). Audio-visual perception of everyday natural objects hemodynamic studies in humans (J. Kaiser \& M. J. Naumer, Eds.). https://doi.org/10.1007/978-1-4419-5615-6

Lewkowicz, D. J., \& Ghazanfar, A. A. (2009). The emergence of multisensory systems through perceptual narrowing. Trends in Cognitive Sciences, 13(11), 470-478. https://doi.org/10.1016/j.tics.2009.08.004

Lord, C., Risi, S., Lambrecht, L., Cook Jr., E. H., Leventhal, Bennett, L., DiLavore, P.C., Pickles, A., Rutter, M. (2000). The Autism Diagnostic Observation Schedule - Generic: A standard mesure of social and communication deficits associated with the spectrum of autism. Journal of Autism and Developmental Disorders, 30(3), 205-223.

https://doi.org/10.1023/A:1005592401947

Magnée, M. J. C. M., de Gelder, B., van Engeland, H., \& Kemner, C. (2011). Multisensory integration and attention in autism spectrum disorder: evidence from event-related potentials. PloS One, 6(8), e24196. 
https://doi.org/10.1371/journal.pone.0024196

McKay, L. S., Simmons, D. R., McAleer, P., Marjoram, D., Piggot, J., \& Pollick, F. E. (2012). Do distinct atypical cortical networks process biological motion information in adults with Autism Spectrum Disorders? Neurolmage, 59(2), 1524-1533. https://doi.org/10.1016/j.neuroimage.2011.08.033

Megnin, O., Flitton, A., Jones, C. R. G., de Haan, M., Baldeweg, T., \& Charman, T. (2012). Audiovisual speech integration in autism spectrum disorders: ERP evidence for atypicalities in lexical-semantic processing. Autism Research : Official Journal of the International Society for Autism Research, 5(1), 3948. https://doi.org/10.1002/aur.231

Mottron, L. (2004). Matching Strategies in Cognitive Research with Individuals with High-Functioning Autism: Current Practices, Instrument Biases, and Recommendations. Journal of Autism and Developmental Disorders, 34(1), 19-27. https://doi.org/10.1023/B:JADD.0000018070.88380.83

Noel, J. P., De Niear, M. A., Stevenson, R., Alais, D., \& Wallace, M. T. (2017). Atypical rapid audio-visual temporal recalibration in autism spectrum disorders. Autism Research, 10(1), 121-129. https://doi.org/10.1002/aur.1633

Noel, J. P., De Niear, M., Van Der Burg, E., \& Wallace, M. T. (2016). Audiovisual simultaneity judgment and rapid recalibration throughout the lifespan. PLoS ONE, 11(8), 1-14. https://doi.org/10.1371/journal.pone.0161698

Nomi, J. S., \& Uddin, L. Q. (2015). Developmental changes in large-scale network connectivity in autism. Neurolmage: Clinical, 7, 732-741. 
https://doi.org/10.1016/j.nicl.2015.02.024

Pierce, K., Müller, R. a, Ambrose, J., Allen, G., \& Courchesne, E. (2001). Face processing occurs outside the fusiform "face area" in autism: evidence from functional MRI. Brain : A Journal of Neurology, 124(Pt 10), 2059-2073.

Retrieved from http://www.ncbi.nlm.nih.gov/pubmed/11571222

Russo, N., Mottron, L., Burack, J. A., \& Jemel, B. (2012). Parameters of semantic multisensory integration depend on timing and modality order among people on the autism spectrum: evidence from event-related potentials. Neuropsychologia, 50(9), 2131-2141. https://doi.org/10.1016/j.neuropsychologia.2012.05.003

Russo, Natalie, Foxe, J. J., Brandwein, A. B., Altschuler, T., Gomes, H., \& Molholm, S. (2010). Multisensory processing in children with autism: highdensity electrical mapping of auditory-somatosensory integration. Autism Research : Official Journal of the International Society for Autism Research, 3(5), 253-267. https://doi.org/10.1002/aur.152

Schwarzlose, R. F., Baker, C. I., \& Kanwisher, N. (2005). Separate face and body selectivity on the fusiform gyrus. Journal of Neuroscience, 25(47), 11055-11059. https://doi.org/10.1523/JNEUROSCI.2621-05.2005

Stein, B. E., Stanford, T. R., \& Rowland, B. A. (2009). The neural basis of multisensory integration in the midbrain: Its organization and maturation. Hearing 2, 258(1-2), 4-15. https://doi.org/10.1016/j.heares.2009.03.012 Stevenson, R.A, Siemann, J. K., Schneider, B. C., Eberly, H. E., Woynaroski, T. G., Camarata, S. M., \& Wallace, M. T. (2014). Multisensory Temporal 
Integration in Autism Spectrum Disorders. Journal of Neuroscience, 34(3), 691-697. https://doi.org/10.1523/JNEUROSCI.3615-13.2014

Stevenson, Ryan A., Baum, S. H., Segers, M., Ferber, S., Barense, M. D., \& Wallace, M. T. (2017). Multisensory speech perception in autism spectrum disorder: From phoneme to whole-word perception. Autism Research, 10(7), 1280-1290. https://doi.org/10.1002/aur.1776

Stevenson, Ryan A., \& Wallace, M. T. (2013). Multisensory temporal integration: task and stimulus dependencies. Experimental Brain Research, 227(2), 249-261. https://doi.org/10.1007/s00221-013-3507-3

Stickel, S., Weismann, P., Kellermann, T., Regenbogen, C., Habel, U., Freiherr, J., \& Chechko, N. (2019). Audio-visual and olfactory-visual integration in healthy participants and subjects with autism spectrum disorder. Human Brain Mapping, 40(15), 4470-4486. https://doi.org/10.1002/hbm.24715 Taylor, P. A., Chen, G., Glen, D. R., Rajendra, J. K., Reynolds, R. C., \& Cox, R. W. (2018). FMRI processing with AFNI: Some comments and corrections on "Exploring the Impact of Analysis Software on Task fMRI Results" Paul A. Taylor, Gang Chen, Daniel R. Glen, Justin K. Rajendra, Richard C. Reynolds, Robert W. Cox. BioRxiv, April.

Thye, M. D., Bednarz, H. M., Herringshaw, A. J., Sartin, E. B., \& Kana, R. K. (2016). The impact of atypical sensory processing on social impairments in autism spectrum disorder. Developmental Cognitive Neuroscience, (May), 0-1. https://doi.org/10.1016/j.den.2017.04.010 Unruh, K. E., Martin, L. E., Magnon, G., Vaillancourt, D. E., Sweeney, J. A., \& 
Mosconi, M. W. (2019). Cortical and subcortical alterations associated with precision visuomotor behavior in individuals with autism spectrum disorder. Journal of Neurophysiology, 122(4), 1330-1341. https://doi.org/10.1152/jn.00286.2019

Vossel, S., Geng, J. J., \& Fink, G. R. (2014). Dorsal and ventral attention systems: Distinct neural circuits but collaborative roles. Neuroscientist, 20(2), 150-159. https://doi.org/10.1177/1073858413494269

Watanabe, M., \& Munoz, D. P. (2011). Probing basal ganglia functions by saccade eye movements. European Journal of Neuroscience, 33(11), 20702090. https://doi.org/10.1111/j.1460-9568.2011.07691.x

Wechsler, D. (2011). Wechsler Abbreviated Scale of Intelligence - second edition (WASI-II). San Antonia, TX: Pearson.

Weiner, K. S., \& Grill-Spector, K. (2011). Not one extrastriate body area: Using anatomical landmarks, hMT+, and visual field maps to parcellate limbselective activations in human lateral occipitotemporal cortex. Neuroimage, 56(4), 2183-2199. https://doi.org/10.1016/j.neuroimage.2011.03.041 
Table 1. Group Demographic and Behavioral Data: The groups are matched for age, gender, handedness, and non-verbal IQ (Perceptual Reasoning). TD-typically developed; ASD-Autism Spectrum Disorder; IQ-Wechsler Abbreviated Scale of Intelligence Composite scores; SP-Adolescent/Adult Sensory Profile; ADOSAutism Diagnostic Observation Schedule (ADOS-2), Module $4(n=17)$, Module 3 $(n=3)$, RRB-Restricted Repetitive Behavior, CSS-Calibrated Severity Score. Data are two-tailed $t$-tests assuming equal variances except where Levene's is significant $\left({ }^{\dagger} p<0.05\right)$, Pearson Chi-Square for Handedness; or Kruskal-Wallis [ $\left.{ }^{*}\right]$ for non-normally distributed data as determined by Shapiro-Wilk test.

\begin{tabular}{|c|c|c|c|c|c|c|c|c|c|}
\hline & \multicolumn{4}{|c|}{ TD Group } & \multicolumn{5}{|c|}{ ASD Group } \\
\hline & Range & $n$ & Mean & $\pm \mathrm{SD}$ & Range & $n$ & Mean & $\pm \mathrm{SD}$ & $t$-test \\
\hline Age (years) & $11-28$ & $21(6 \mathrm{~F})$ & 20.1 & 4.4 & $12-27$ & $20(5)$ & 18.7 & 4.5 & $P=0.31$ \\
\hline Handedness (right/left) & na & $21 / 0$ & na & na & na & $18 / 2$ & na & na & $P=0.14$ \\
\hline IQ-Perceptual Reasoning & $88-127$ & $19(6 \mathrm{~F})$ & 110 & 12.4 & $75-140$ & $20(5)$ & 107 & 16.7 & $P=0.56$ \\
\hline IQ-Verbal & $95-160$ & $19(6 \mathrm{~F})$ & 117 & 14.6 & $74-134$ & $20(5)$ & 102 & 17.2 & $P=0.006$ \\
\hline FSIQ-4 & $90-135$ & $19(6 \mathrm{~F})$ & 115 & 11 & $75-133$ & $20(5)$ & 105 & 16.5 & $P=0.037$ \\
\hline fMRI Total Task Errors & $0-6$ & $20(5 F)$ & 1.7 & 1.6 & $0-40$ & $20(5)$ & 8.1 & 11.9 & $P=0.15^{*} \dagger$ \\
\hline Total Omissions & $0-4$ & $20(5 \mathrm{~F})$ & 1.0 & 1.0 & $0-8$ & $20(5)$ & 1.6 & 2.4 & $P=0.31^{*} \dagger$ \\
\hline Total Commissions & $0-3$ & $20(5 \mathrm{~F})$ & 0.7 & 0.9 & $0-39$ & $20(5)$ & 6.5 & 10.5 & $P=0.02^{*} \dagger$ \\
\hline Omissions-V & $0-2$ & $20(5 \mathrm{~F})$ & 0.35 & 0.6 & $0-5$ & $20(5)$ & 1.0 & 1.6 & $P=0.22^{*} \dagger$ \\
\hline Commissions-V & $0-2$ & $20(5 \mathrm{~F})$ & 0.40 & 0.6 & $0-17$ & $20(5)$ & 3.3 & 5.0 & $P=0.04^{*} \dagger$ \\
\hline Omissions-AV & $0-3$ & $20(5 \mathrm{~F})$ & 0.65 & 0.9 & $0-4$ & $20(5)$ & 0.5 & 1.1 & $P=0.38^{*}$ \\
\hline Commissions-AV & $0-2$ & $20(5 F)$ & 0.30 & 0.6 & $0-22$ & $20(5)$ & 3.3 & 5.6 & $P=0.02^{*} \dagger$ \\
\hline$\%$ TRs Censored for Motion & $0-7 \%$ & $21(6 \mathrm{~F})$ & 1.04 & 2.11 & $0-14 \%$ & $20(5)$ & 2.65 & 4.27 & $P=0.08^{*} \dagger$ \\
\hline SP-Low Registration & $16-35$ & $14(3 F)$ & 28 & 5.6 & $26-50$ & $19(5 \mathrm{~F})$ & 38 & 7.1 & $P<0.001$ \\
\hline SP-Sensation Seeking & $41-59$ & $14(3 \mathrm{~F})$ & 49 & 5.5 & $31-57$ & $19(5 \mathrm{~F})$ & 41 & 6.6 & $P=0.001$ \\
\hline SP-Sensory Sensitivity & $18-51$ & $14(3 F)$ & 32 & 10.4 & $28-63$ & $19(5 F)$ & 39 & 10.5 & $P=0.05^{*}$ \\
\hline ADOS RRB & na & na & na & na & $1-10$ & $20(5)$ & 6.8 & 2.5 & na \\
\hline ADOS CSS & na & na & na & na & $5-10$ & $20(5)$ & 7.6 & 1.7 & na \\
\hline
\end{tabular}


Table 2 Regions of Interest from (A) within-group contrasts (TD audio-visual [AV] minus TD visual-only [V]; ASD AV minus ASD V) and (B) between-group contrasts (ASD AV minus TD AV; ASD V minus TD V). ROls are derived in AFNI (3dMVM) thresheld at $t=3.56, p=0.001$, nearest neighbor 2 (NN2), Bisided for all ROls. Minimum cluster sizes based on output from 3dClustSim. ROI volumes were used to generate ROI masks from which BOLD PSC values were extracted from each subject, group averaged, and subsequently t-tested (two-tailed paired for within-group contrasts and two-tailed unpaired for between-group contrasts). ASD $n=20$ and TD n=21. AV - audio-visual, V - visual-only, ASD - Autism Spectrum Disorder, TD - Typically Developed, $d$ - Cohen's $d$, BOLD - Blood Oxygen-Level Dependent, PSC - Percent Signal Change, SC superior colliculus, GP - globus pallidus, STG - superior temporal gyrus.

\begin{tabular}{|c|c|c|c|c|c|c|c|c|c|c|c|c|c|c|c|c|c|c|c|c|c|c|c|c|c|c|c|}
\hline & \begin{tabular}{|l} 
Contrast for \\
Derivation of \\
ROls
\end{tabular} & \begin{tabular}{|r|} 
Min \\
Clust \\
alpha
\end{tabular} & $\begin{array}{l}\text { Min } \\
\text { Clust } \\
\text { p }\end{array}$ & $\begin{array}{c}\text { Min } \\
\text { Cluster } \\
\text { Vol }\end{array}$ & alph & Volume & Woxels & $\mathrm{CM}_{2}$ & CM & $\mathrm{CMz}$ & Peak & Peak & Peak: & ROI & Hemi & & \begin{tabular}{|l|} 
Mean \\
BOLD \\
Vis-only \\
TD \\
\end{tabular} & $\begin{array}{l}\text { Mean } \\
\text { BOLD } \\
\text { AV TD }\end{array}$ & $t$-test & 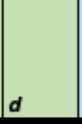 & \begin{tabular}{|l|} 
Mean \\
BOLD \\
Vis-only \\
ASD \\
\end{tabular} & \begin{tabular}{|l|} 
Mean \\
BOLD \\
AV ASD
\end{tabular} & $t$-test & $d$ & \begin{tabular}{|l|} 
Vis-only \\
$t$-test \\
ASD vs \\
TD \\
\end{tabular} & $\left|\begin{array}{l}\text { AV } t \text {-test } \\
\text { ASD vs TD }\end{array}\right|$ & $d$ \\
\hline & TD AV - TD V & $<0.01$ & 0.001 & 51 & $<0.01$ & 21952 & 512 & 53.2 & \begin{tabular}{|l|}
-19.9 \\
\end{tabular} & 9.0 & 66.5 & -21.5 & 18.0 & STG & Right & $A \bigvee>V$ & 0.010 & 0.331 & $3.8 \mathrm{E}-12$ & -1.72 & 0.002 & 0.280 & $2.6 \mathrm{E}-08$ & -1.92 & $8.7 \mathrm{E}-01$ & $3.6 \mathrm{E}-01$ & na \\
\hline & TD AV-TD V & $<0.01$ & 0.001 & 51 & $<0.01$ & 18651 & 435 & -49.7 & -25.5 & 111.1 & -66.5 & -14.5 & 11.0 & STG & Left & $A V>V$ & 0.061 & 0.389 & $8.9 \mathrm{E}-10$ & -1.69 & 0.020 & 0.355 & \begin{tabular}{|l|}
$3.1 \mathrm{E}-07$ \\
\end{tabular} & -1.56 & $4 \mathrm{E}-01$ & $5 \mathrm{E}-01$ & na \\
\hline & TD AV-TDV & $<0.01$ & 0.001 & 51 & $<0.01$ & 2916 & 68 & -3.8 & -33.8 & -4.4 & -14.0 & -28.5 & -6.5 & 3 Pulvinar/SC & Bilateral & $A V>V$ & -0.020 & 0.171 & \begin{tabular}{|l|}
$1.6 \mathrm{E}-07$ \\
\end{tabular} & -1.08 & 0.004 & 0.107 & $1.3 \mathrm{E}-02$ & \begin{tabular}{|l|} 
\\
\end{tabular} & $6 \mathrm{E}-01$ & $4 \mathrm{E}-01$ & na \\
\hline & ASD AV - ASD V & $<0.01$ & 0.001 & 48 & $<0.01$ & 18994 & 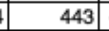 & $\begin{array}{ll}-50.8 \\
\end{array}$ & \begin{tabular}{|c|}
-26.4 \\
\end{tabular} & 11.1 & -42.0 & -35.5 & 14.5 & $4 \longdiv { \text { STG } }$ & Left & $A V>V$ & 0.070 & 0.385 & $5.0 \mathrm{E}-10$ & -1.56 & 0.013 & 0.370 & \begin{tabular}{|l|}
$2.4 \mathrm{E}-07$ \\
\end{tabular} & $|-1.64|$ & $2.9 \mathrm{E}-01$ & $8.5 \mathrm{E}-01$ & na \\
\hline & ASD AV - ASD V & $<0.01$ & 0.001 & 48 & $<0.01$ & 17407 & \begin{tabular}{l|l}
406 & \\
\end{tabular} & 52.4 & \begin{tabular}{|c|}
-18.3 \\
\end{tabular} & 8.4 & 66.5 & -11.0 & 7.5 & $5 \longdiv { \text { STG } }$ & Right & $A V>V$ & 0.000 & 0.348 & \begin{tabular}{|l|}
$2.7 \mathrm{E}-12$ \\
\end{tabular} & -1.72 & -0.007 & 0.320 & $2.3 \mathrm{E}-08$ & \begin{tabular}{|l|}
3.05 \\
\end{tabular} & $9 \mathrm{E}-01$ & $.4 \mathrm{E}-01$ & na \\
\hline 3. & ASD - TD Vis-only & 0.05 & 0.001 & 28 & 0.05 & 1201 & 28 & -27.0 & -0.2 & -2.4 & -24.5 & -0.5 & -6.5 & Putamen/GP & Left & $T D>A S D$ & 0.273 & 0.246 & \begin{tabular}{|l|}
$2.2 \mathrm{E}-01$ \\
\end{tabular} & na & 0.067 & 0.062 & $8.3 \mathrm{E}-01$ & na & $3.3 \mathrm{E}-05$ & $1.4 \mathrm{E}-04$ & 1.48 \\
\hline & ASD - TD Vis-only & 0.05 & 0.001 & 28 & 0.05 & 1201 & 28 & -33.9 & \begin{tabular}{|c|}
-47.7 \\
\end{tabular} & \begin{tabular}{l|l|l|}
46.6 & -1
\end{tabular} & -31.5 & -49.5 & 49.5 & $7 \longdiv { \text { IPS } }$ & Left & $T D>A S D$ & 0.265 & 0.222 & $2.9 \mathrm{E}-02$ & & -0.015 & -0.043 & $3.1 \mathrm{E}-01$ & 7 & $5.5 \mathrm{E}-06$ & $3.6 \mathrm{E}-06$ & $\Rightarrow$ \\
\hline
\end{tabular}


Figure 1. fMRI Task. While in the MRI participants watched a continuous video of a person bouncing a basketball filmed from the shoulders down to avoid confounds of facial processing. Task blocks were either Audio-visual (AV) or Visual-only (V). Participants wore ear buds to hear the video. A simple attention task involved pressing a button with their left index finger when they perceived the ball to hit the floor. The video lasted approximately 5 minutes with the task blocks (10 sec each) pseudo-randomly distributed between rest blocks (10 sec each) during which the actor stood still holding the ball. Two runs were created in which the task blocks were counter-balanced: Run A: AV-AV-V-AV-V-V-AV-V-V-AV-AV-AV-V-V; Run B: V-V-AV-V-AV-AV-V-AV-AV-V-V-V-AV-AV). Each run was presented twice (Run A, B, A, B). Button presses were continuously monitored throughout and used to calculate task errors (omissions and commissions). There were 14 task blocks in each run with either 8 or 9 bounces in each 10 sec task block totaling 119 bounces per run for a grand total of 476 basketball bounces across the four video runs. Each presentation of the video began with a black screen with the words "GET READY" in white letters. Each video run ended with the words "KEEP HOLDING STILL" in white letters on a black background to remind the participants that they could not move even though the video had ended.

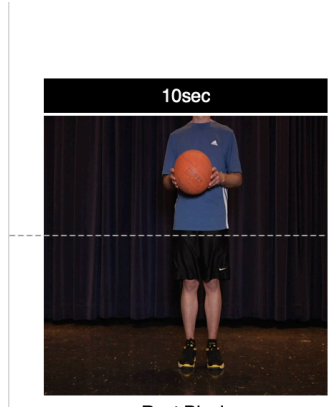

Rest Block

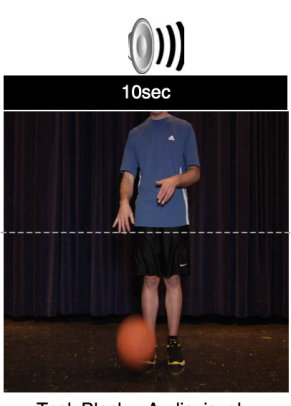

Task Block - Audiovisual

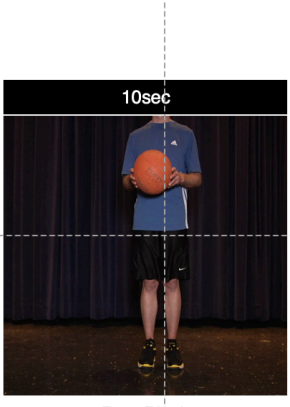

Rest Biock

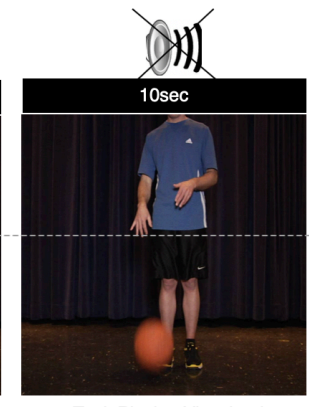

Task Block - Visual-only

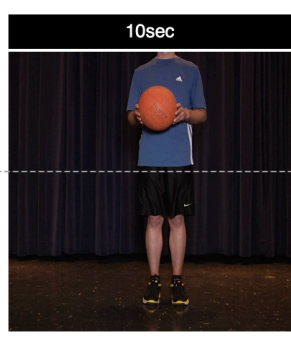

Rest Block 
Figure 2. Within-group whole-brain contrasts between the conditions. (A) ASD audio-visual (AV) minus ASD visual-only (V). (B) TD AV minus TD V. (C) The TD group also showed AV > V (yellow) in bilateral pulvinar/superior colliculus while no other ROIs survived thresholding and cluster correction for the ASD group. All ROIs are thresheld at $t=3.56, p=0.001$ and cluster corrected at $\alpha<0.01, p=0.001$ (Table 2). Data are in MNI space and projected onto the MNI152 template. ROI volumes were used to make ROI masks from which BOLD PSC values were extracted from each subject and group averaged to create the histograms (twotailed $t$-tests of group averaged BOLD in each ROI with: ${ }^{*} p<0.05,{ }^{* * *} p<0.001$. ASD-Autism Spectrum Disorder, TD-typically developed, SC-superior colliculus, STG-superior temporal gyrus; BOLD-Blood Oxygen-Level Dependent signal; ROIregion of interest; \% = PSC, Percent Signal Change. 

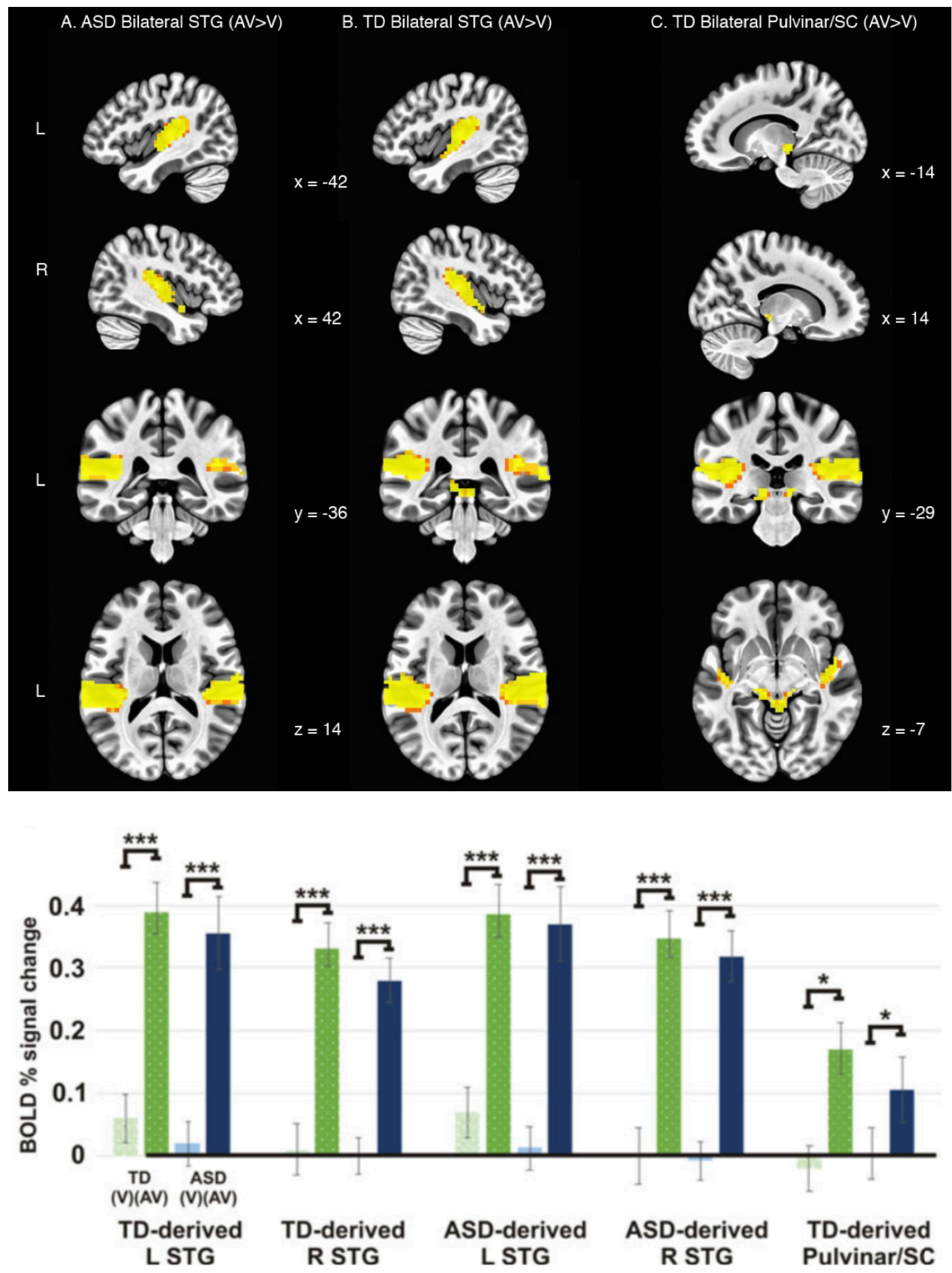
Figure 3. Between-group whole-brain contrast of group averaged BOLD PSC values for the visual-only condition (ASD visual-only minus TD visual-only). BOLD hypoactivation (blue) is seen in the ASD group compared to the TD group in the (A) left putamen/GP and (B) left intraparietal sulcus. Data are thresheld at $t=3.56 p=0.001$ for all ROls and cluster corrected at $\alpha=0.05, p=0.001$. Data are in $\mathrm{MNI}$ space and projected onto the MNI152 template. ROI volumes were used to make ROI masks from which BOLD PSC values were extracted from each subject and group averaged to create the histograms (two-tailed $t$-tests of group averaged BOLD in each ROI with ${ }^{* * *} p<0.001$ and $d>1.3$ for all ROIs). ASDAutism Spectrum Disorder, GP-globus pallidus, TD-typically developed, BOLDBlood Oxygen-Level Dependent, ROI-region of interest, PSC-Percent Signal Change. 
$\mathrm{L}$

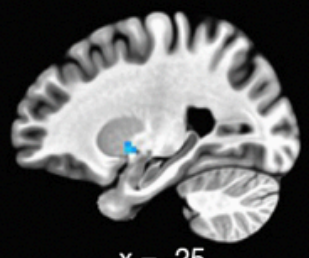

$x=-25$
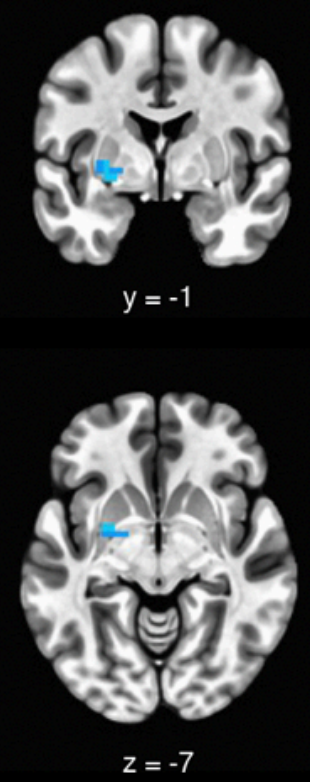

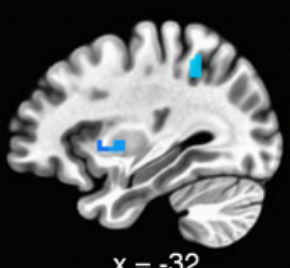

$x=-32$
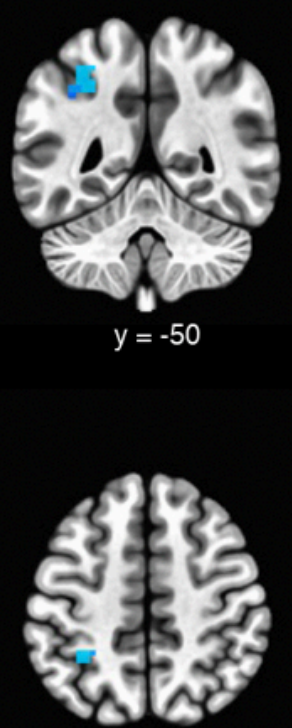

$z=49$

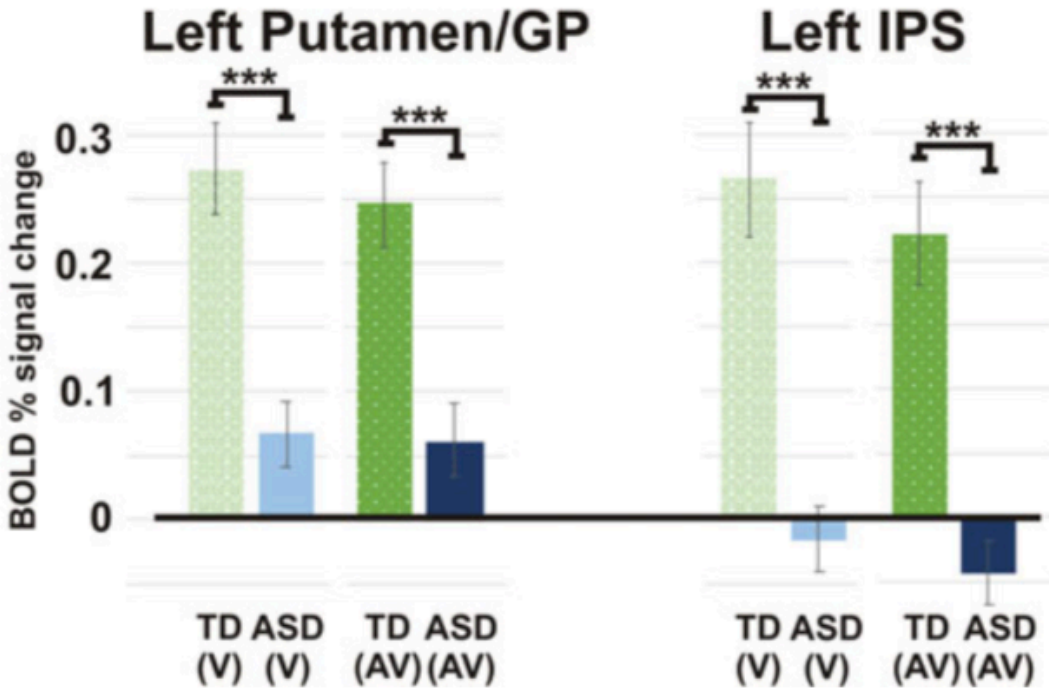




\section{Chapter III}

\section{GENERAL DISCUSSION}

Brief Summary

Multisensory integration is an experience-dependent ability that begins to develop in infancy and facilitates how we perceive and move through the world. When brain function and/or structure are aberrant, this can impact our capacity to integrate multiple sensory inputs which in turn can alter developmental trajectories of language, motor coordination, and communication-all core deficits in ASD. Since input from one sensory modality can alter our perception of incoming information from other senses, neuroimaging studies investigating sensory processing from a multisensory perspective are critical to elucidating underlying brain mechanisms that contribute to the behavioral phenotypes of ASD.

The use of stimuli in neuroimaging studies that most closely reflect the real world may facilitate the identification of functional and structural brain differences in individuals with ASD for integrating complex, socially relevant sensory information from their environment. In the present study, we used well-controlled dynamic natural stimuli to examine neural differences in audio-visual multisensory integration in ASD. Contrary to our hypotheses, we observed hypoactivation in the left IPS and left putamen/GP in the ASD group. The purpose of this section is to provide additional thoughts on these findings and then propose future directions 
for additional studies that were not included in the publication. First, we discuss the implications of group differences and then discuss possible future studies controlling for stimulus characteristics to further elucidate underlying mechanisms that may be contributing to these group differences.

Inferences for the Primary Results of this Study

In the present study, the use of real-world dynamic stimuli revealed hypoactivation in the corticobasal loop (IPS - putamen/GP; Figure 3) in the ASD group compared to controls. These ROls were revealed solely in the visual-only condition and not in the audio-visual condition based on a whole-brain analysis. The lack of the identification of ROIs with a significant group difference in the audiovisual condition might suggest that the addition of auditory sensory input in the audio-visual (multisensory) condition may have facilitated brain activation levels in the ASD group such that those with ASD no longer differed from controls. However, the group averaged BOLD activation levels extracted from these ROls (Figure 3 histogram) tell a different story. The ASD group showed statistically lower extracted BOLD levels compared to controls within these ROls in both conditions. While there was no interaction between group and condition, there was a main effect of group for both conditions in these ROls. This suggests that the addition of multisensory processing did not differentially impact the hypoactivation in the IPS-putamen/GP circuit. Thus, it appears that the deficit is at the basic processing level for unisensory (visual) information processing. To confirm this, we would 
need to examine the impact of the addition of auditory information and determine if there is a similar hypoactivation to auditory information only, which would require a different experimental paradigm.

One possible explanation for the discrepancy may be due to the whole-brain fMRI analysis used to derive the ROIs. This method requires correction for multiple comparisons across $\sim 100 \mathrm{k}$ voxels in the brain. Data from this stringent whole-brain method for identifying ROIs would benefit from significantly increasing the number of participants in the ASD group along with decreasing the heterogeneity in this group. The BOLD data extracted for each condition in the ROI masks generated from the visual-only group contrast show that this group difference was consistent across both conditions. A difference between the groups from the extracted BOLD data is supported by the continual commission task errors by the ASD group across both conditions. There is no change in BOLD data and no change in behavior. Thus, while no ROI group differences resulted from the whole brain between group analysis in the audio-visual condition, the group did show a consistent hypoactivation in this brain region from the extracted BOLD data for both conditions, which is supported by their commission task errors that also persisted regardless of condition.

The lack of a change in BOLD activation levels within either group for the IPS and putamen/GP ROls would indicate that these brain regions were not influenced by the addition of auditory information in the audio-visual condition and thus not likely involved in integrating auditory and visual inputs to process these stimuli or to perform the task. Although caution is needed in drawing inferences 
about negative data, the hypoactivation in the left IPS in the ASD group may reflect differences in function or structure possibly involving (1) biological motion processed in the left IPS from the left EBA/FBA; (2) structural connections from left EBA/FBA to the left IPS; (3) functional motor upper limb coordination from right IPS to left IPS; or (4) structural interhemispheric connections from right IPS to left IPS. Possible explanations of this hypoactivation are important for the development of future studies. Below we propose future studies that test each of the above possible underlying mechanisms that may be contributing to the results in the present study.

\section{Future Studies}

In the present study the ASD group consistently demonstrated hypoactivation in the left IPS and putamen/GP ROls compared to TD controls. Thus, increasing activation in this brain region would be a likely goal of a targeted intervention for ASD as has been done in prior studies (Calderoni et al., 2016). However, first the contributions of the task and stimuli would need to be delineated in future studies to better understand the possible functional and structural influences on this hypoactivation. Based on the above hypotheses for this hypoactivation, we propose the following:

1) Biological motion processed in the left IPS from the left EBA/FBA: In order to determine whether hypoactivation in the IPS is a reflection of 
processing of biological motion stimuli from the EBA and/or FBA, the task could be altered to remove the person in the scene and just include the ball bouncing at the same rhythm. In this way, we could remove the contribution of biological motion processing and isolate the contribution of the visual search task itself while maintaining the complexities of coordinating the button press for the visuomotor task.

2) Structural connections from left EBA/FBA to left IPS: In the present study, no group differences were found in the EBA or FBA brain regions as a result of the whole-brain group analysis. Therefore, it is possible the ASD group was using the EBA/FBA similarly to controls. Thus, the hypoactivation in the left IPS may reflect altered white matter connections between the left EBA and IPS and/or between the left FBA and IPS. These white matter tracts include the inferior longitudinal fasciculus and arcuate fasciculus (Gschwind, Pourtois, Schwartz, Van De Ville, \& Vuilleumier, 2012). A DTI analysis of these white matter tracts would provide insight into the structural integrity of these tracts and further delineate the contributions of function and structure for processing biological motion stimuli and the IPS hypoactivation observed in the ASD group. DTI measures of structural integrity could be correlated with measures of sensory processing to delineate the contributions of these white matter tracts in processing this stimulus. 
3) Functional motor upper limb coordination from right IPS to left IPS: Using the stimulus as they were delivered in the present study, but without a motor response, would remove the potential contribution of interhemispheric motor coordination on the BOLD levels in the IPS. This also may indicate whether the hypoactivation in the IPS is feeding forward to impact BOLD levels in the putamen/GP for processing of the visuomotor button press task.

4) Structural interhemispheric connections from right IPS to left IPS: As in \#(2) above, using DTI to investigate the structural integrity of the white matter interhemispheric connections between the left and right IPS (splenium and midbody of the corpus callosum) would delineate whether hypoactivation in the left IPS may be the result of structural differences.

In addition, to these proposed studies, there are a number of modifications to the stimuli that may provide clarity to the interpretation of our findings. For example, considering the similar BOLD activation in both groups for both conditions, using the same sound stimuli from the present study and creating an auditory-only task would help to determine if the unisensory and multisensory findings are specific to visual stimuli or also pertain to auditory stimuli.

Using eye-tracking data to monitor attention to the stimuli would assist interpretation of all of the above proposed future studies, by removing the need for a motor response. In addition, combining fMRI and EEG to localize functional 
aspects of multisensory processing and simultaneously capture temporal dynamics (respectively) of multisensory processing may better elucidate the underlying brain mechanisms of these findings. Few studies examine both aspects simultaneously as the compounding effects of sensory inputs from the testing equipment (wearing an EEG cap while in an MRI) may be excessive for some individuals on the autism spectrum. However, examining both aspects simultaneously would provide a unique advantage to capture temporal and functional aspects of sensory integration to more accurately assess patterns of sensory integration (Mantini, Marzetti, Corbetta, Romani, \& Del Gratta, 2010).

While the use of dynamic natural stimuli has the added benefit of capturing sensory integration processes that reflect the real-world, these stimuli can be modified to make them more well-controlled creating quasi-natural stimuli that could facilitate the delineation of specific underlying neural mechanisms for processing real-world dynamic stimuli. Below we outline a few ways in which this can be done.

Use of Dynamic Natural Stimuli The use of dynamic natural stimuli in neuroimaging research investigating multisensory integration is necessary to explore the complex interactions in the human brain when processing multiple sensory inputs simultaneously; however, this type of stimuli poses significant challenges to neuroimaging research due to the inherent complexities of a continuously moving scene (Eg \& Behne, 2015; Rummukainen, Radun, Virtanen, \& Pulkki, 2014; Vodrahalli et al., 2018). These difficulties are compounded by the 
need to incorporate an attention task in order to ensure that participants are observing the stimuli over long periods of time. Therefore, recommendations for future research here consider the contributions of task difficulty and stimulus complexity.

Since biological motion stimuli are known to be processed in very discrete brain regions (extrastriate body area and fusiform body area (Grossman \& Blake, 2002)), much like human vocalizations and human faces, future studies using dynamic, real-world stimuli should take into account the increased complexity of combining multiple sensory inputs with biological motion stimuli. One way to disentangle processing of these various components would be to use a real-world stimulus that moves and has sound but does not involve biological motion. For example, one study tested motion perception using the forced-choice motion discrimination task in which participants view a group of dots moving in a given direction at varying coherence levels based upon the proportion of dots moving in a specific direction (low=10\% to high-80\%) (Robertson, Martin, Baker, \& Baron-Cohen, 2012). Participants were instructed to consider the task as if the dots were leaves and they were detecting the direction of the wind. This well-suited analogy for the task could be developed to incorporate a real tree with leaves that move using computer animation to obtain the desired coherence levels enabling the testing of a realworld stimulus in this task, but with well-controlled elements of motion. These types of visual motion stimuli would not be confounded with human biological motion processing and yet would be natural and dynamic. 
In the present study, a simple task of attention proved to be critical for assuring that participants attended to the stimuli throughout the duration of the video; however, this fMRI data in the present study would have benefited from eyetracking data. In the above-mentioned examples of possible natural stimuli that do not contain biological motion, it may be difficult to identify an attention task that does not involve participants counting. Thus real-world videos may need to incorporate computer-generated aspects that look realistic and facilitate participant attention while being timed to capture BOLD activation. For example, if a dynamic video of the ocean tide is used, a fish or other sea animal may appear in the water or on the shore that would naturally be found in that setting. Thus the combination of dynamic, real-world stimuli with well-controlled computer-generated aspects that appear real may facilitate detection of more fine-grained aspects of sensory processing and integration while avoiding confounds inherent in stimuli that incorporate human biological motion, vocalizations, and faces.

While neuroimaging studies investigating multisensory integration in ASD are able to elucidate underlying neural mechanisms that contribute to the behavioral phenotypes used to diagnose and treat ASD, the outcomes of these studies have been difficult to integrate into a coherent theory of multisensory integration in ASD. This is due to several factors including the inclusion of children and adults in the same data sets considering that multisensory integration ability improves with age, the different levels of difficulty in attention tasks or the absence of an attentional measure to ensure engagement, and the level of complexity of the stimuli (natural versus nonnatural and linguistic vs nonlinguistic). 
Task-based sensory integration studies that incorporate multiple neuroimaging methods have the greatest chance of characterizing underlying functional and structural contributions to their findings. These methods include DTI (structural connectivity), task-based fMRI (good spatial resolution), resting state fMRI (functional connectivity), and EEG (good temporal resolution). For example, one study that was previously mentioned used DTI to investigate structural white matter connections between regions of interest revealed in their fMRI analysis (Sahyoun et al., 2010). The DTI data enhanced the interpretation of the functional ROI group differences found in this study; however, combining imaging techniques means that participants will undergo testing for longer periods of time. Thus considerations for the length of time in an MRI and the added sensory input of additional neuroimaging testing must be considered especially when conducting research with individuals having varied sensory differences. Data processing methods that can reduce the time needed in an MRI along with behavioral methods to help these participants hold still and maintain their attention will help to facilitate these studies.

Lastly, our understanding of sensory processing in ASD would benefit from the inclusion of individuals who may be more affected by their ASD. One recent study (Gabrielsen et al., 2018) was successfully able to use fMRI to investigate brain function while children and adolescents with low verbal and cognitive performance watched a video of their choosing. Enabling broader participation would improve the generalization of neuroimaging studies investigating sensory processing to the broader autism spectrum providing a more meaningful 
interpretation of the results that can be extrapolated for the development of interventions and to elucidate the developmental trajectories of ASD.

\section{Final Conclusions}

Sensory processing differences are highly prevalent in ASD and are thought to underlie other core deficits in this disorder; therefore, gaining a better understanding of the neural mechanisms that underly sensory deficits in ASD is critical to better understand the etiology of this disorder. The present study utilized fMRI to characterize neural mechanisms in ASD when processing dynamic, realworld audio-visual stimuli. The results demonstrated that adolescents and adults with ASD do not differ from controls in auditory and visual cortical and subcortical brain regions, but surprisingly they showed hypoactivation in corticobasal brain regions that process and coordinate visuo-motor input. Taken together, this data implicates higher-order processing of biological motion stimuli and possible deficits in visuo-motor disinhibition. Future studies are needed to further characterize the functional and/or structural brain mechanisms that may be contributing to these deficits. 


\section{References}

Calderoni, S., Billeci, L., Narzisi, A., Brambilla, P., Retico, A., \& Muratori, F. (2016). Rehabilitative interventions and brain plasticity in autism spectrum disorders: Focus on MRI-based studies. Frontiers in Neuroscience, 10(MAR), 1-7. https://doi.org/10.3389/fnins.2016.00139

Eg, R., \& Behne, D. M. (2015). Perceived synchrony for realistic and dynamic audiovisual events. Frontiers in Psychology, 6(June). https://doi.org/10.3389/fpsyg.2015.00736

Gabrielsen, T. P., Anderson, J. S., Stephenson, K. G., Beck, J., King, J. B., Kellems, R., ... South, M. (2018). Functional MRI connectivity of children with autism and low verbal and cognitive performance. Molecular Autism, 9(1), 1-14. https://doi.org/10.1186/s13229-018-0248-y

Grossman, E. D., \& Blake, R. (2002). Brain areas active during visual perception of biological motion. Neuron, 35(6), 1167-1175. https://doi.org/10.1016/S0896-6273(02)00897-8

Gschwind, M., Pourtois, G., Schwartz, S., Van De Ville, D., \& Vuilleumier, P. (2012). White-matter connectivity between face-responsive regions in the human brain. Cerebral Cortex, 22(7), 1564-1576.

https://doi.org/10.1093/cercor/bhr226

Mantini, D., Marzetti, L., Corbetta, M., Romani, G. L., \& Del Gratta, C. (2010). Multimodal integration of $\mathrm{fMRI}$ and EEG data for high spatial and temporal resolution analysis of brain networks. Brain Topography, 23(2), 150-158. 
https://doi.org/10.1007/s10548-009-0132-3

Robertson, C. E., Martin, A., Baker, C. I., \& Baron-Cohen, S. (2012). Atypical Integration of Motion Signals in Autism Spectrum Conditions. PLOS ONE, 7(11), 1-9. https://doi.org/10.1371/journal.pone.0048173

Rummukainen, O., Radun, J., Virtanen, T., \& Pulkki, V. (2014). Categorization of natural dynamic audiovisual scenes. PLoS ONE, 9(5), 1-14. https://doi.org/10.1371/journal.pone.0095848

Sahyoun, C. P., Belliveau, J. W., Soulières, I., Schwartz, S., \& Mody, M. (2010). Neuroimaging of the functional and structural networks underlying visuospatial vs. linguistic reasoning in high-functioning autism. Neuropsychologia, 48(1), 86-95. https://doi.org/10.1016/j.neuropsychologia.2009.08.013

Vodrahalli, K., Chen, P. H., Liang, Y., Baldassano, C., Chen, J., Yong, E., Honey, C., Hasson, Uri, Ramadge, P., Norman, K.A., Arora, S. (2018). Mapping between $\mathrm{fMRI}$ responses to movies and their natural language annotations. Neurolmage, 180(1), 223-231. https://doi.org/10.1016/j.neuroimage.2017.06.042 
Appendix 1 Raven's Progressive Matrices

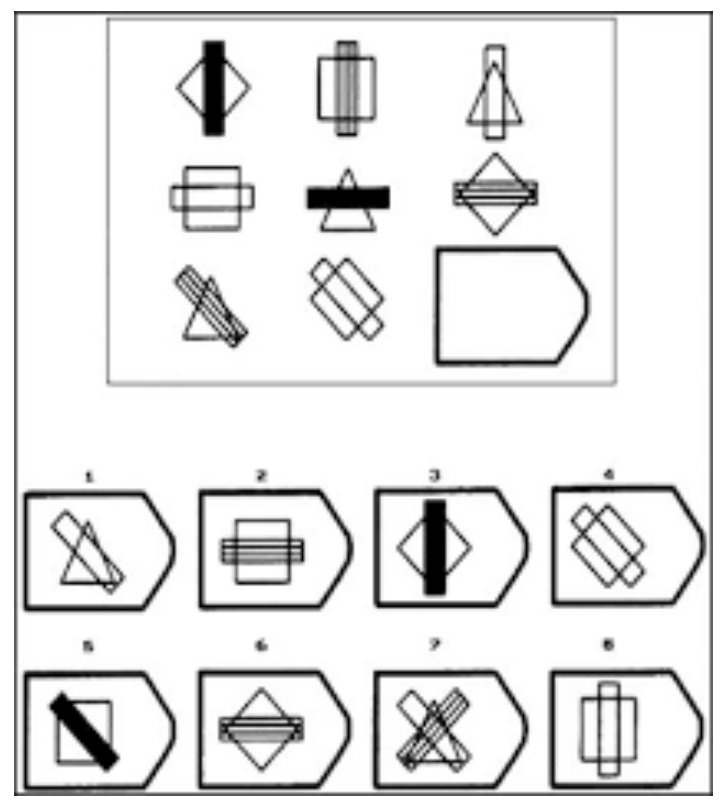

